\title{
LA PROGENÈSE CHEZ LES TRÉMATOdES DIGÉNÉTIQUES (suite)
}

\author{
Recherches personnelles sur deux espèces progenétiques \\ déjà connues : Ratzia joyeuxi (E. Brumpt, 1922) \\ et Pleurogenes medians (Olsson, 1876)
}

\section{Par Alice BUTTNER}

\section{B. Ratzia joyeuxi (E. Brumpt, 1922)}

C'est en 1919, qu'E. Brumpt trouva chez des Discoglossus pictus (Otth.), du Jardin d'Essais d'Alger, des kystes sous-cutanés contenant chacun un distome dont l'utérus était parfois rempli d'œufs. Les discoglosses parasités furent conservés en captivité à Paris, au laboratoire de parasitologie de la Faculté de Médecine, et Brumpt constata que les distomes enkystés grandissaient en même temps que leur kyste, tandis que leurs ébauches organiques, en particulier les glandes génitales, continuaient à se développer.

Les œufs, de plus en plus nombreux et pourvus d'un miracidium, se répandaient dans le kyste, qui prenait alors une coloration brunâtre. Au bout de quelques semaines, tous les kystes avaient une taille d'environ $1 \mathrm{~mm}$.; le distome libéré atteignait 3 à $4 \mathrm{~mm}$., sa différenciation organique et son développement paraissaient achevés. Puis, ces vers dégénéraient sur place dans leur kyste bourré d'œufs, que l'on retrouvait disséminés dans les tissus voisins, ou bien ils en sortaient spontanément et tombaient dans le milieu extérieur. On les voyait pendant quelques heures se mouvoir au fond de l'aquarium, puis mourir. Les métacercaires observées chez ces discoglosses devenaient toutes progénétiques.

Ces renseignements ont été relevés dans les protocoles expérimentaux d'E. Brumpt, et dans le résumé qu'en donne R.-Ph. Dollfus dans son travail de 1929 sur Ratzia parva (Stossich 1904). Dans la troisième édition de son Précis de Parasitologie (1922), Brumpt a considéré les animaux sortant de leur kyste comme des distomes adultes (1). Il leur a donné le nom d'Opisthorchis (Brachymetra)

(1) Dans la $3^{e}$ édition de son Précis de Parasitologie (1922, p. 176), E. Brumpt écrit : « R.-Ph. Dollfus désigne sous le nom de « métacercaires progénétiques » les métacercaires qui continuent leur évolution dans le kyste en montrant un

Ann. de Parasitologie, T. XXVI, N ${ }^{\circ}$ 3. -1951. 


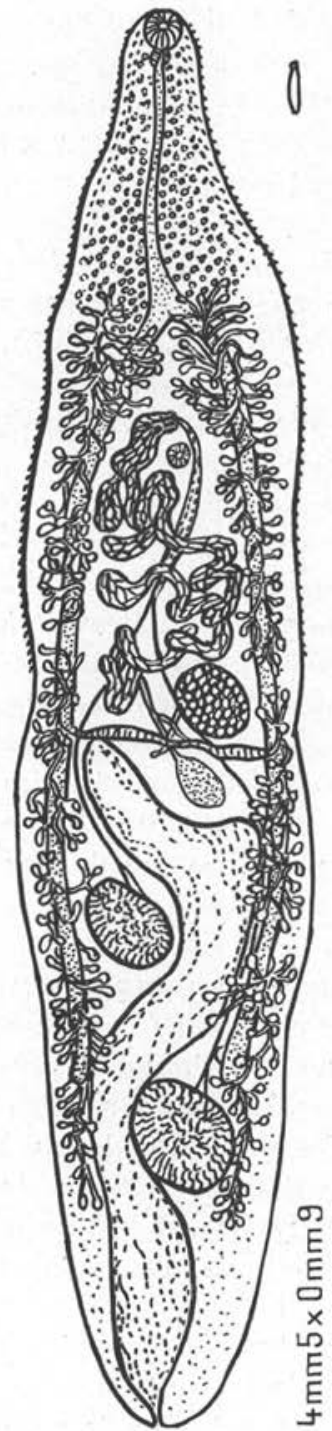

FIti. 46. - Ratzia joyeuxi (Brunipt, 1922) (d'après B. Brumpt). joyeuxi Brumpt 1922. D'accord avec R.-Ph. Dollfus, E. Brumpt a fait entrer ensuite cette espèce dans le genre Ratzia F. Poche 1926.

\section{Observations expérimentales Discussion sur la morphologie de ce distome}

Depuis la découverte de ces métacercaires en 1919 (fig. 46), E. Brumpt a eu souvent l'occasion de revoir ces distomes sur des discoglosses expédiés d'Alger par Ed. Sergent et L. Parrot. C'est ainsi qu'il a pu étudier certains infusoires propres à ces batraciens et découvrir deux nouveaux trypanosomes, Trypanosoma sergenti et $T$. parroti, qui n'ont pu être transmis par Helobdella algira, parasites habituels de Discoglossus pictus Otth. Ces discoglosses ayant pondu à diverses reprises, dans les aquariums qui les contenaient, les têtards éclos ont été nourris avec la chair d'une vingtaine de discoglosses, préalablement autopsiés, dont la peau avait été arrachée. Signalons dès à présent qu'au cours de ces autopsies, des métacercaires n'ont jamais été trouvées dans les muscles de ces discoglosses.

Les kystes de Ratzia joyeuxi varient du blanc au noir, selon leur degré de maturité. Les kystes blanes ont en moyenne $660 \mu$; les œufs bruns sont embryonnés dans l'utérus et mesurent $34-39 \mu$ de long sur 20-23 $\mu$ de large ; 50 p. 100 de ces métacercaires ont déjà des œufs évacués dans le kyste et tournent assez vite à l'intérieur de leur enveloppe.

Les kystes noirs tombent à la maturité ; ils se gonflent dans l'eau et, en deux à cinq heures, les trématodes sortent, puis meurent rapidement. Chaque jour, chez les dis-

développement sexuel précoce ; certaines peuvent même atteindre l'état adulte et pondre de nombreux œufs embryonnés sans changer d'hôte. 》 E. BRUMPT donne une figure (fig. 334, p. 176) que nous avons reproduite (Ann. P'aras., XXV, 1950,388 , fig. 8) d'un disceglosse mort le 12 juin, très parasité, et où l'on voit de nombreux distomes sortir de leur kyste sur l'animal. 
coglosses très parasités, il tombe environ un kyste qui s'ouvre normalement ; en général, la localisation est sous-cutanée : sur environ 200 kystes trouvés chez un discoglosse, 194 se trouvaient sous l'épiderme ; 6 étaient localisés dans le tissu conjonctif ou dans les aponévroses sous-cutanées ; il n'y en avait point dans les viscères, ni dans la cavité péritonéale.

Le 24 mai 1919, le contenu d'un bocal, où quatre à cinq kystes, lors de la déhiscence, avaient laissé échapper leurs œufs, a été versé par E. Brumpt dans une cuve d'élevage de Limnæa palustris. Le 4 juin, les jeunes limnées se développaient normalement; malheureusement, elles succombèrent accidentellement quelques jours plus tard, et l'évolution chez d'autres mollusques ne put être reprise.

A cette époque, en effet, E. Brumpt ne possédait pas d'élevages neufs des prosobranches les plus connus en France, Vivipara, Valvata, Bithynia, chez lesquels il aurait pu tenter d'élucider le cycle. C'est pourquoi, il avait choisi des Limnæa palustris, dont il avait à ce moment un certain nombre d'exemplaires neufs. Nous discuterons cette question plus loin et montrerons qu'un Opisthorchïdæe avait peu de chances d'évoluer chez un mollusque pulmoné. Le 12 juin, 26 kystes furent prélevés chez un discoglosse pour étudier l'évolution des œufs. Ces œufs furent exposés pendant plus d'une heure, dans la matinée, à un soleil très chaud; aucune éclosion ne fut observée et cependant les miracidiums étaient bien formés et vivants dans la coque.

En 1923, Ch. Joyeux trouvait chez des grenouilles (Rana esculenta L., var. ridibunda Pallas), de l'oasis de Gafsa, des petits kystes intramusculaires répandus dans tout le corps. Ces Rana hébergeaient ce parasite dans la proportion de 2 sur 7 . Dans un autre travail, Joyeux signale n'avoir trouvé ces kystes que dans le tissu musculaire ; " ils sont arrondis, noirs, possèdent environ $800 \mu$ de diamètre. La paroi kystique a $25 \mu$ d'épaisseur ; elle est entourée d'une zone de faible réaction, formée par le tissu musculaire condensé de l'hôte ».

Joyeux note encore que tous les trématodes examinés en septembre sont plus ou moins dégénérés; les vitellogènes sont difficilement visibles, les testicules et l'ovaire dégénèrent; les œufs sont très abondants ; ils mesurent $35 \mu$ sur $17 \mu$ et sont en grande partie répandus dans le kyste. Dans bien des cas, ils ont été ingérés par l'animal après leur évacuation et remplissent les cæcums digestifs (fig. 47). Le distome est parfois complètement dégénéré et seuls les œufs restent disséminés dans les tissus.

En 1927, Ch. Joyeux revient sur l'étude de cette métacercaire qu'il identifie à $R$. parva (Stossich); il écrit : «Ce parasite a été décrit par Stossich chez des grenouilles de l'Italie orientale. Il forme 

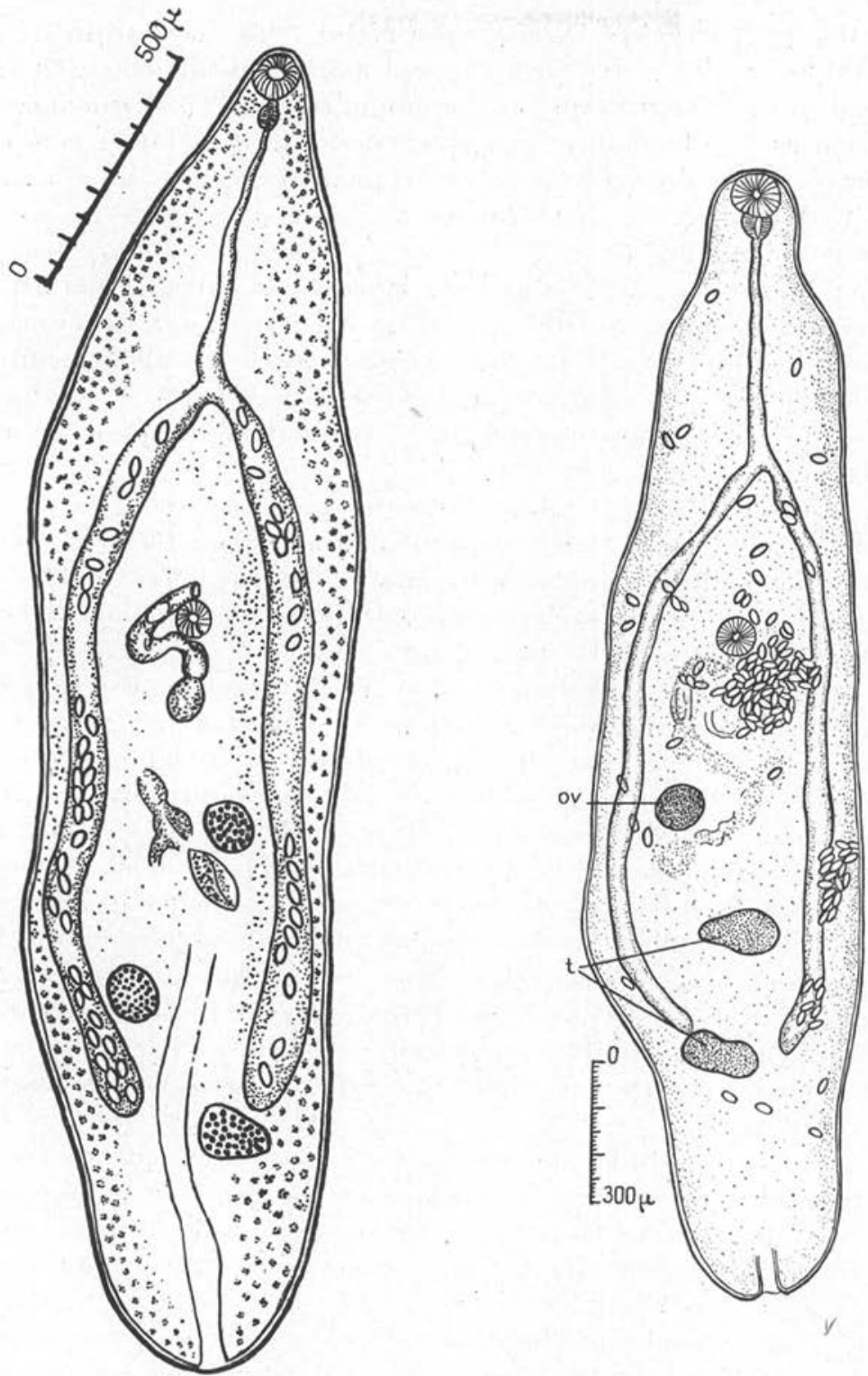

Fig. 47. - Métacercaires progénétiques intramusculaires de Fiano esculenta, var. ridibunda de Gafsa, identifiées à $R$. parva (Stoss.) par Ch. Joyeux (dessins de préparations de Ch. Joyeux). (Orig.).

des kystes intramusculaires, à l'intérieur desquels se trouve le trématode. Je l'ai retrouvé chez $R$. esculenta L., var. ridibunda, à Gafsa, 
1923. D'autre part, chez Discoglossus pictus Otth., du Jardin d'Essais à Alger, on rencontre fréquemment des kystes analogues signalés et figurés par E. Brumpt sous le nom d'Opisthorchis (Brachymetra) joyeuxi. R.-Ph. Dollfus, qui a eu l'occasion d'examiner comparativement les préparations de ces trématodes, pense qu'il s'agit d'une seule espèce, $R$. parva (Stossich), ayant la priorité au point de vue nomenclature. »

Joyeux ne trouve pas de différence appréciable entre les parasites de Rana esculenta et de Discoglossus pictus d'une part, la description donnée par Stossich de Brachymetra parva. en 1904, d'autre part. Il signale seulement, ce que Stossich n'avait pas remarqué, qu'il existe un volumineux réceptacle séminal situé au-dessus de l'ovaire.

Néanmoins, cet auteur relève, entre les métacercaires progénétiques de discoglosses et celles des Rana de Gafsa, une différence biologique importante : l'habitat sous-cutané des premières, et l'habitat intramusculaire des métacercaires de Rana esculenta de Gafsa, parasitées dans la proportion de 2 sur 7 .

En octobre 1926, cependant, Joyeux relate que des discoglosses récoltés au Jardin d'Essais, présentaient, « chez le même batracien », à la fois des parasites sous-cutanés et intramusculaires. L'auteur ne précise pas s'il a trouvé ces formes intramusculaires chez un ou plusieurs discoglosses, ni leur nombre, et il émet l'hypothèse que, selon la saison, l'enkystement se fait plus ou moins profondément dans les tissus de l'hôte. Rappelons que le Professeur Brumpt nous a confirmé n'avoir jamais trouvé de métacercaires de Ratzia joyeuxi dans les muscles. Nous avons eu nous-même la bonne fortune d'observer chez deux Discoglossus pictus du Jardin d'Essais d'Alger, sur dix-huit exemplaires expédiés, un certain nombre de métacercaires progénétiques de cette même espèce localisées sous la peau.

Par ailleurs, les études que nous avons faites sur l'enkystement d'assez nombreuses espèces de métacercaires nous ont permis de constater que, dans la majeure partie des cas, la localisation des métacercaires est particulière : peau, sous les écailles, branchies, muscles, reins, cavité générale, etc...

Il est donc possible que les kystes trouvés dans les muscles du discoglosse d'Alger par Ch. Joyeux soient des parasites erratiques, comme cela se produit parfois. Observons d'ailleurs que Joyeux a examiné à la même époque neuf Rana esculenta du Jardin d'Essais et les a trouvées toutes négatives, tandis que des discoglosses capturés au même endroit étaient infestés dans la proportion de 7 sur 11.

Ces faits de spécificité parasitaire, concernant à la fois l'hôte et la localisation des métacercaires, nous apparaissent comme une 
première et importante différence biologique, postulant en faveur de la non-identité entre les parasites de Discoglossus pictus et ceux de Rana esculenta.

En octobre 1926 également, Joyeux a examiné 16 têtards d'âges divers du Jardin d'Essais, dont il ne donne malheureusement pas le nom spécifique : ils n'ont présenté aucune métacercaire, de même que deux jeunes discoglosses provenant du même gìte. Pour Joyeux, ces métacercaires de Discoglossus seraient plutôt une infestation de l'àge adulte, opinion qui ne pourra être confirmée ou infirmée que lorsque la cercaire sera connue.

Nous avons autopsié 30 têtards de Discoglossus provenant des " regards » d'irrigation du Jardin d'Essais (1), où ont été capturés les discoglosses parasités que nous avons étudiés ; ils se sont montrés tous négatifs.

Nous avons fréquemment observé, dans le cas des xiphidiocercaires, que lorsqu'un batracien adulte est infesté expérimentalement par des cercaires, il l'est presque toujours par un très petit nombre, tandis que les têtards, beaucoup plus sensibles, succombent en général à une hyperinfestation. S'il en était également ainsi dans le cas des métacercaires progénétiques de discoglosses et de grenouilles, il se pourrait que les têtards infestés soient morts, échappant de la sorte à l'observation, et qu'il ne reste plus que les têtards indemnes. Il se peut aussi que les têtards de discoglosse ne fréquentent pas exactement les mêmes gìtes que l'animal adulte.

R.-Ph. Dollfus (1929) considère les trois distomes progénétiques de Stossich, Brumpt et Joyeux comme identiques, Stossich ayant la priorité pour leur dénomination. Toutefois, le nom générique de $B r a-$ chymetra donné par Stossich, ayant déjà un homonyme chez un genre d'insectes, Brachymetra Mayr, fut abandonné pour celui de Ratzia, la sous-famille devenant ainsi celle des Ratziinæ.

Tout en admettant l'éventualité d'un cycle abrégé chez Ratzia parva, Dollfus estime qu'il n'en existe pas moins un cycle complet comportant un hôte définitif normal hébergeant la véritable forme adulte. Il a recherché cet adulte chez des animaux faisant leur proie de grenouilles et de discoglosses. Dollfus a ainsi découvert, dans l'intestin d'une couleuvre fer à cheval, Zamenis hippocrepis, originaire de Rabat (Maroc), le 22 juillet 1925, trois exemplaires d'un distome adulte qui, "sans contestation possible, était l'état parfait de la métacercaire progénétique des grenouilles et des discoglosses 》.

"Entre le véritable adulte et la métacercaire progénétique (à son

(1) Nous remercions le $\mathrm{D}^{\mathrm{r}} \mathrm{L}$. Parrot, qui nous a procuré ces animaux, de même que nos discoglosses parasités. 
maximum de développement atteint dans le kyste), les différences sont nettes, dit encore cet auteur : elles ne portent pas sur l'ovaire, l'utérus, les vitellogènes, les œufs qui ont même forme et mème

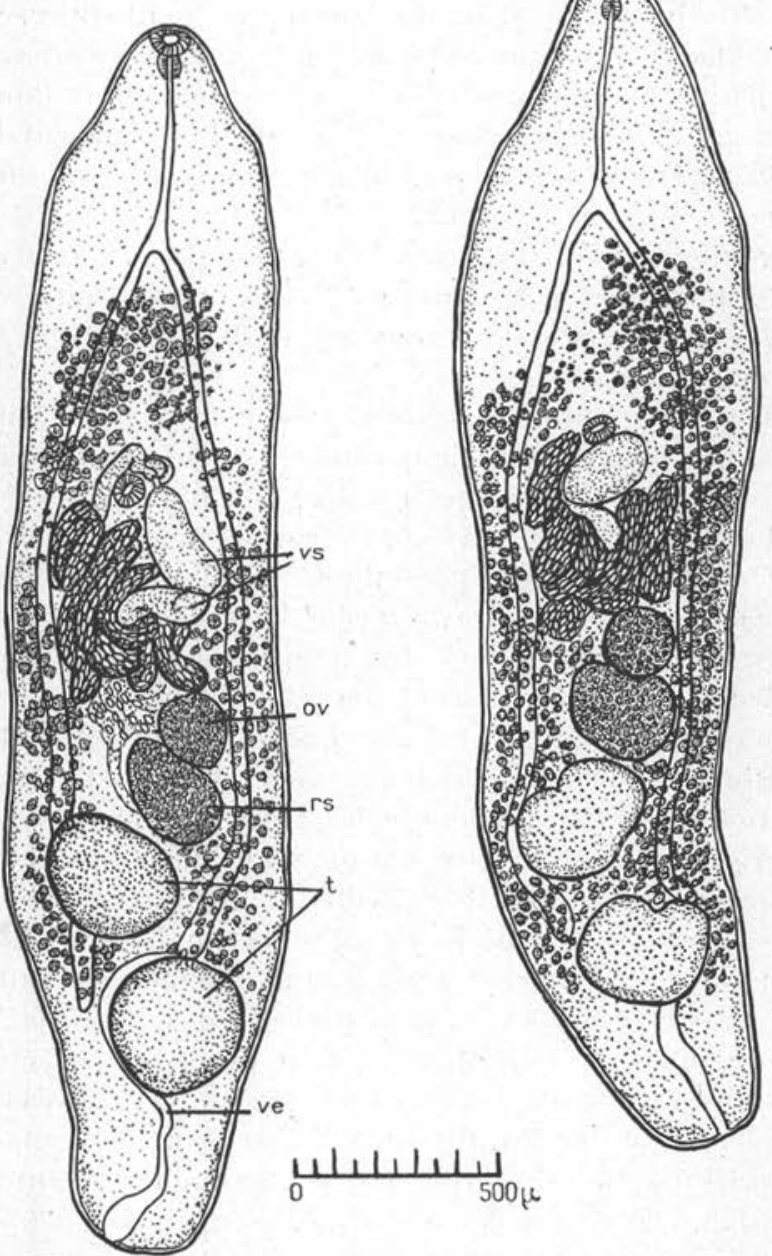

Frg. 48. - Ratzia dollfusi A. Buttner, 1950, trouvées dans l'intestin de Zamenis hippocrepis (Maroc) par R.-Ph. Dollfus ; ov. : ovaire ; r.s.. . réceptacle séminal; $t$.: testicules; ve.: vessie ; $p . s$. : vésicule séminale (dessins orig. de préparations prêtées par R.-Ph. Dollfus).

importance, mais l'on remarque chez l'adulte que le receptaculum seminis est beaucoup plus volumineux et surtout qu'il existe une vésicule séminale externe bipartite très importante, libre dans le parenchyme, précédant la portion terminale de l'appareil mâle ; 
cette dernière portion consiste en une sorte de manchon sacciforme (prostatique ?), entourant le canal déférent jusqu'à l'orifice génital. Il existe des différences de moindre importance, par exemple les ćpines cuticulaires de la métacercaire ne persistent pas et la cuticule de l'adulte n'est plus ornée que d'une très fine ponctuation ; la vessie, très large chez la métacercaire, perd son ampleur chez l'adulte. »

Grâce à l'amabilité de R.-Ph. Dollfus, nous avons pu avoir en main les préparations in toto des Ratzia trouvées par lui chez Zamenis hippocrepis (fig. 48). Nous avons pu les comparer avec nos propres préparations de Ratzia joyeuxi et les coupes de ses kystes.

Nous donnons, pour permettre leur comparaison, différentes figures de ces quatre types de distomes (voir figs 5, 46, 47, 48 et 49).

On est immédiatement frappé par la ressemblance qui existe entre les métacercaires progénétiques décrites par Stossich et celles trouvées par Joyeux dans les muscles de grenouilles vertes : mêmes proportions et même disposition des ventouses, l'acetabulum étant un peu au-dessus du plan transversal médian du distome. Les testicules et l'ovaire sont peu développés ; la vessie tubuliforme passe entre les testicules. Les préparations dont nous disposions, bien que pâlies, nous ont permis de distinguer la volumineuse vésicule séminale dont parle Ch. Joyeux, mais non les vitellogènes. L'identité de ces deux distomes nous paraît très probable, tant au point de vue morphologique que biologique.

Signalons cependant qu'une métacercaire progénétique, trouvée par L. Balozet chez Rana ridibunda, dans le Sud tunisien (Oued Bezik), et dont il a bien voulu nous communiquer récemment la préparation (fig. 49), présente de grandes analogies avec les métacercaires de discoglosse : la vessie est volumineuse et semblable de forme ; les organes génitaux ont la même disposition.

Il reste, pour les différencier, l'habitat de ces métacercaires dans des tissus distincts.

Mais nous ne partageons pas l'opinion de R.-Ph. Dollfus en ce qui concerne l'identité de Ratzia joyeuxi (Brumpt 1922) et des Ratzia adultes de Zamenis hippocrepis.

Notons en effet que :

- Les glandes vitellogènes sont très développées chez $R$. joyeuxi ; elles s'étendent, en avant, au-dessus de la bifurcation des cæcums digestifs ; postérieurement, elles dépassent le niveau du dernier testicule. Chez Ratzia sp. de Zamenis hippocrepis, elles n'atteignent pas la division des cæcums et s'arrêtent, en général, au bord supérieur du dernier testicule.

- Ratzia joyeuxi possède une vessie volumineuse, bien visible et constante chez toutes les métacercaires de discoglosses étudiées 
par nous; chez les Ratzia trouvés dans l'intestin de Zamenis, on la distingue à peine.

- Les testicules de $R$. joyeuxi sont généralement sphériques, et le tractus génital mâle est entièrement développé ; néanmoins, il ne semble pas fonctionnel, ainsi que nous le montrerons plus loin, en décrivant le développement de l'œuf chez cette métacercaire. Cette carence fonctionnelle des testicules existe d'ailleurs également chez les métacercaires progénétiques de Rana ridibunda. Les glandes génitales mâles des Ratzia de la couleuvre fer à cheval sont au contraire énormes, lobées, et en pleine activité génitale; $\mathrm{de}$ nombreux spermatozoïdes sont présents dans la vésicule séminale et le receptaculum seminis de cette dernière espèce. Dans ces conditions, il devient difficile de comprendre comment une métacercaire de Discoglossus pictus ou de Rana esculenta, dont les testicules, même chez les métacercaires jeunes, sont en pleine dégénérescence, peut re-

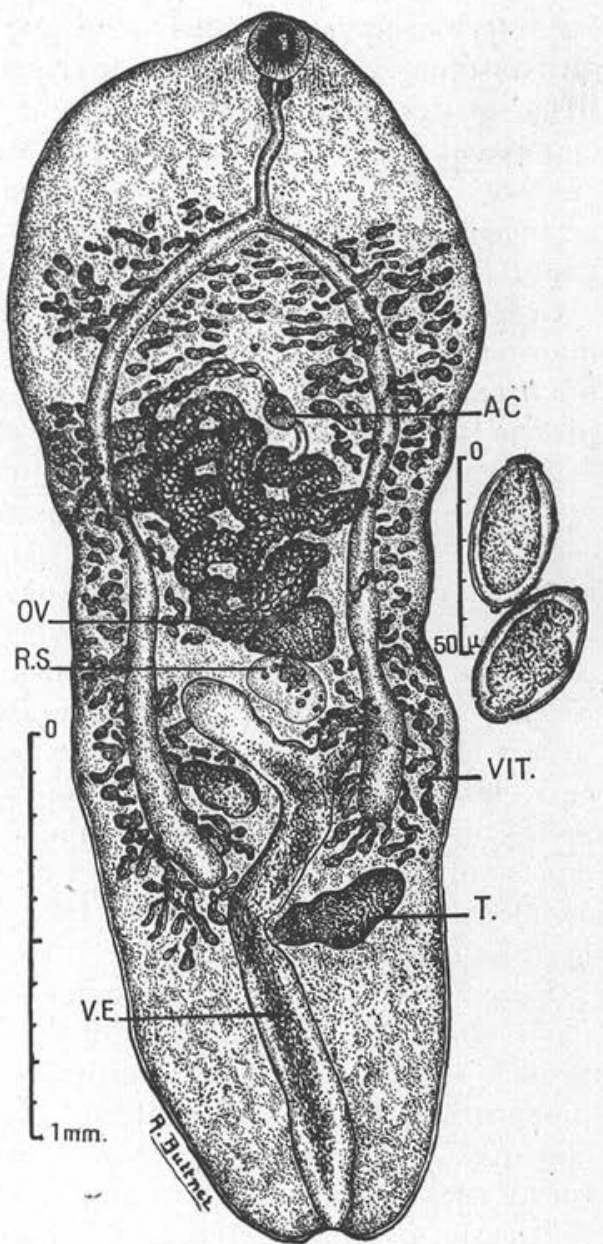

Fic. 49. - Métacercaire progénétique identifiée à Ratzia parioa; trouvée chez Rana ridibunda de l'Oued Bezik (Suci tunisien). AC. : acetabulum ; OV. : ovaire ; R.S.: réceptacle séminal; T. : testicule; V.E. : vessie : VIT. : glandes vitellogènes. (Dessin original d'une préparation de L. Balozet).

trouver une activité génitale normale par simple passage chez un hôte définitif.

Il nous parait donc, biologiquement et morphologiquement, plus rationnel d'admettre ici, jusqu'à preuve expérimentale du contraire, trois espèces différentes de Ratzia : 
- Ratzia parva (Stossich 1904), retrouvée par Ch. Joyeux, parasite de Rana esculenta ridibunda, enkystée dans les muscles, normalement progénétique.

- Ratzia joyeuxi (Brumpt 1922), parasite de Discoglossus pictus et enkystée normalement sous la peau, toujours progénétique.

- Ratzia.n. sp., distome à évolution inconnue, parasite à l'état adulte de Zamenis hippocrepis, dont le cycle doit comporter certainement toujours trois hôtes, et que nous désignerons sous le nom de Ratzia dollfusi n. sp.

\section{Etude de l'évolution. Essais de cycle abrégé}

Nous ignorons à quelle époque se fait l'infestation des discoglosses ; un fait certain, c'est que, dès fin mars, on trouve chez ces animaux des métacercaires jeunes qui mûrissent rapidement et atteignent, dans des conditions de laboratoire, leurs dimensions maximum fin juin. A cette époque, beaucoup de kystes tombent naturellement et on peut apercevoir des métacercaires sortant spontanément de leurs kystes, soit au fond de l'aquarium, soit même sur l'animal vivant. Leur sortie est beaucoup plus rapide lorsqu'un. discoglosse très parasité meurt.

Ces documents semblent indiquer que la métacercaire peut devenir tout à fait adulte en trois à quatre mois au maximum, puisque nos préparations du 9 mai sont des distomes parfaitement mûrs. Cependant, même sous le climat d'Alger, Joyeux a trouvé, fin octobre, 7 discoglosses sur 11 présentant dans leurs téguments des métacercaires mûres de Ratzia joyeuxi. Peut-être l'infestation par les cercaires ne s'est-elle produite que fin juillet.

Les œufs de ces métacercaires mûres ont-ils un miracidium et peuvent-ils infester directement les mollusques ?

A propos de la maturité des œufs, Joyeux admet que l'œuf se développe en culture ; en cultivant, en boîte de Pétri, à Gafsa, en juillet-août 1932, des œufs de Ratzia parva intramusculaíre de $R$. esculenta, var. ridibunda, il a constaté la formation du miracidium au bout de quatre jours.

A Paris déjà, pendant l'été 1930, des œufs avaient été mis en culture le $1^{\text {er }}$ août ; ils ont mûri en une quinzaine de jours, mais l'auteur ne dit pas si les miracidiums ont éclos dans le milieu extérieur ; il ne spécifie pas non plus s'il s'agit d'œufs de parasites de Rana ou de Discoglossus, bien qu'il paraisse vraisemblable qu'à cette époque, ce fussent plutôt de métacercaires de $D$. pictus.

Signalons, d'autre part, qu'en octobre 1926, en utilisant des kys- 
tes intramusculaires, Joyeux a assisté à l'éclosion des miracidiums dans l'eau au bout d'une heure.

Cette éclosion des miracidiums au contact de l'eau est un fait assez exceptionnel dans le cas des Opisthorchiidæ. E. Brumpt ne l'a pas observé chez $R$. joyeuxi, malgré l'exposition d'œufs contenant un miracidium au soleil chaud de juin. Nous-même, pour étudier éventuellement l'attraction miracidienne de certains prosobranches, avons exposé, sans plus de succès, pendant plus de deux heures, de nombreux œufs embryonnés de $R$. joyeuxi au soleil et à la lumière de Wood.

On ne connait guère qu'une observation analogue, non confirmée par d'autres chercheurs, relatée dans les études du savant japonais M. Muto (1918), sur le cycle évolutif d'un autre Opisthorchiidæ, très commun en Extrême-Orient chez l'homme et divers mammifères, Clonorchis sinensis (Cobbold 1873), dont le miracidium éclôt, d'après cet auteur, dans le milieu extérieur. Il évolue chez le mollusque prosobranche Bithynia striatula, var. japonica Pilsburg, et donne des oculolophocercaires qui s'enkystent exclusivement sous les écailles de certains poissons, dont le plus important est le vulgaire poisson rouge (Carassius auratus), souvent consommé cru.

Cependant, dans des expériences ultérieures, Faust et Khaw (1927) précisent que les œufs sont "ingérés 》 par les Bithynia et ils donnent à ce sujet un schéma caractéristique. D'autre part, le Professeur H. Galliard, qui a fait également, à Hanoï, des recherches sur l'évolution de Clonorchis sinensis, nous a signalé (communication verbale) qu'il n'a jamais assisté à l'éclosion des miracidiums.

Habituellement, en effet, chez les espèces d'O pisthorchiidæ à évolution connue, les miracidiums éclosent dans le tube digestif du mollusque-premier hôte intermédiaire (voir tableau) :

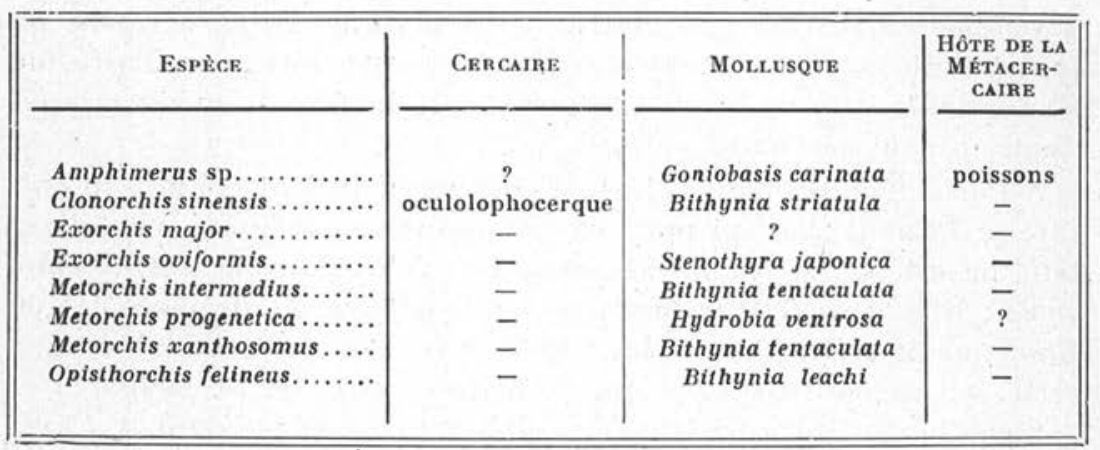


On ne connaît malheureusement pas la cercaire qui infeste les discoglosses d'Algérie. S'il s'agit bien d'un Opisthorchiidæx typique, il est probable que cette cercaire est une lophocercaire ocellée, évoluant normalement chez des mollusques prosobranches, dont les représentants les plus fréquents en Afrique du Nord sont les genres Melania, Melanopsis, Cleopatra, etc...

Joyeux, Rondeau du Noyer et Baer (1930) ont essayé sans succès de faire évoluer les miracidiums éclos chez Bithynia tentaculata, Limnæa limosa, Planorbis corneus L., Physa subopaca Lam. et Limnæa truncatula Müll.; ces quatre dernières espèces de mollusques sont des pulmonés ; 155 Physa subopaca récoltées le 18 février, au Jardin d'Essais d'Alger, se sont montrées indemnes d'infestation. Les auteurs n'ont pas observé si ces divers mollusques exerçaient une attraction spéciale sur les miracidiums.

Le 24 avril, nous avons tenté d'infester également une vingtaine de Bithynia tentaculata jeunes d'élevage avec les œufs embryonnés d'une métacercaire progénétique de Discoglossus pictus du Jardin d'Essais d'Alger ; nous n'avons retrouvé que peu d'œufs parmi les déjections de ces mollusques et aucune coque désoperculée permettant de préjuger de leur éclosion dans l'intestin : ces mollusques ne se sont pas infestés.

Nous avons fait un essai également avec de jeunes Melanopsis originaires de Biskra (Algérie), mais nous n'avons pas réussi non plus à les infester.

Nous avons essayé d'obtenir des mollusques prosobranches, originaires du Jardin d'Essais d'Alger; malheureusement, il faut croire que ces animaux n'apparaissent que tardivement, car les seuls mollusques qui ont pu être récoltés dans les canaux d'irrigation, en mars, avril et mai, sont des physes, certainement tout à fait étrangères à ce cycle évolutif.

Désirant vérifier l'hypothèse de Dollfus, selon laquelle les métacercaires progénétiques de grenouilles et de discoglosses sont identiques aux trois spécimens trouvés par lui chez Zamenis hippocrepis (L.), Joyeux et ses collaborateurs (1930) ont fait ingérer à une couleuvre à collier, Tropidonotus natrix, des environs de Dijon, de nombreux kystes de Discoglossus pictus d'Alger. L'autopsie, effectuée au bout de trois jours, montre que les trématodes retrouvés dans l'intestin n'ont pas évolué et semblent plutôt dégénérés.

Recommençant l'expérience, ils infestent quatre Tropidonotus natrix et une Coluber æsculapi Lacépède, avec une grande quantité de kystes de discoglosses :

- une $T$. natrix et la $C$. æsculapi sont sacrifiées au bout de quatre jours et trouvées indemnes de parasites; 
— une T. natrix, sacrifiée le $12^{\circ}$ jour, est également indemne de trématodes ;

- une T. natrix, sacrifiée le $14^{\circ}$ jour, est encore négative ;

— enfin, la dernière $T$. natrix, également tuée le $14^{\circ}$ jour, pré.. sente un seul exemplaire de Ratzia dans son intestin. Ce trématode mesure $4 \mathrm{~mm}$. de long sur $1 \mathrm{~mm}$. de large, c'est-à-dire les dimensions présentées par les métacercaires de Discoglossus pictus ; sa taille ne s'est donc aucunement accrue; en outre, on observe la dégénérescence des organes génitaux.

En octobre et novembre 1929, les auteurs ont eu l'occasion d'examiner deux jeunes Zamenis du Jardin d'Essais d'Alger, dont l'intestin ne renfermait pas de trématodes.

Les insuccès de ces auteurs leur font émettre l'hypothèse que, peut-être, l'infestation ne se produit que chez Zamenis hippocrepis, à l'exclusion des autres reptiles. Puis, Joyeux modifia son opinion et, en 1943, signalant la persistance possible, à l'état de vie ralentie, de certains trématodes chez un hôte anormal, survivance suivie de dégénérescence ou d'expulsion du ver, il conclut : "Nous pensons donc que Ratzia parva est un parasite des batraciens, chez lesquels il arrive normalement à l'état adulte; il peut persister quelque temps dans l'intestin des couleuvres, mais n'y accomplit aucune évolution.»

Cet auteur avait, en effet, trouvé chez une Tropidonotus viperinus Boie, originaire de Tozeur, deux trématodes qu'il identifie à Ratzia parva (Stoss.), se trouvant depuis neuf jours au moins dans l'intestin de ce reptile, demeuré à jeun pendant cette périołe. Les distomes semblaient encore vivants, mais immobiles.

Ces expériences aboutissent donc aux mêmes résultats que pour Paralepoderma brumpti précédemment étudié : un distome, ayant atteint dans son kyste le développement et la fécondité d'un adulte, paraît avoir achevé son évolution ; il ne gagne vraisemblablement rien à un séjour supplémentaire chez un hôte définitif, sinon peutêtre la mise en liberté des œufs emprisonnés dans le kyste.

Cependant, désireuse de nous rendre compte par nous-même si la couleuvre fer à cheval n'avait pas une réceptivité spéciale à l'égard des métacercaires de Ratzia joyeuxi, nous avons essayé de. faire évoluer un certain nombre de kystes progénétiques de cette espèce chez un Zamenis hippocrepis (L.).

Le $\mathrm{D}^{\mathrm{r}}$ André Maurice (1), Chef de Service de l'Institut d'Hygiène de Rabat, nous a fait parvenir, fin juin 1951, un bel exemplaire de ce colubridé, mesurant $1,10 \mathrm{~m}$. de long.

(1) Nous lui exprimons ici nos plus sincères remerciements. 
Le 26 juin, nous avons fait ingérer à cette couleuvre 12 métacercaires progénétiques de Ratzia joyeuxi, constituant, avec 3 autres métacercaires progénétiques utilisées pour l'infestation de Bithynia tentaculata d'élevage et de Melanopsis, la totalité des kystes présentés par un Discoglossus pictus du Jardin d'Essais d'Alger (exp. 677/A.B.-I).

Neuf de ces kystes provenaient de la peau et trois des aponévroses thoraciques et abdominales de cet animal.

Le 29 juillet, le même Zamenis a ingéré cinq nouvelles métacercaires progénétiques de Ratzia joyeuxi trouvées chez un second Discoglossus pictus de même origine que le premier ; deux de ces métacercaires étaient très évoluées et peut-être même en dégénérescence, car le kyste était entièrement noir (exp. 688/A.B.-I).

En raison du faible nombre de kystes que nous possédions (20 en tout chez 2 discoglosses sur 18 envoyés d'Alger), nous n'avons pas jugé bon d'en fixer et d'en colorer quelques-uns, car nous avions à notre disposition de nombreuses préparations de métacercaires progénétiques de discoglosses de la collection E. Brumpt. Nous le regrettons aujourd'hui, car nous aurions peut-être observé chez l'une ou l'autre de ces métacercaires, contrairement à ce qui se produit habituellement, un processus de fécondation normal qui aurait jeté quelque lumière sur les résultats expérimentaux obtenus ultérieurement chez notre Zamenis.

Le 6 août, ce Zamenis tué à l'éther fut soigneusement autopsié par nous. Le premier lot de métacercaires ingérées par cet animal comptait par conséquent 41 jours de séjour dans le tube digestif, le second lot 7 jours seulement.

Nous avons retrouvé dans l'intestin grêle antérieur et moyen, à l'exclusion de tout autre trématode, trois distomes adultes, très actifs, correspondant aux métacercaires de Ratzia joyeuxi absorbées expérimentalement.

Ces trois trématodes, fixés et colorés in toto à l'hémalun-éosine, avaient chacun, dans la vésicule séminale, une ou deux masses fortement colorées de spermatozoïdes, plus ou moins agglutinés. L'examen au fort grossissement nous a montré chez l'un de ces trois spécimens (représenté fig. 51) deux semblables masses dans les deux poches de la vésicule séminale bipartite observée chez les métacercaires et les adultes du genre Ratzia. Dans la poche supérieure, de longs spermatozoïdes, parfaitement individualisés, s'échappaient de l'agglutinat. La partie terminale du tractus génital mâle laissait encore apparaitre quelques rares spermatozoïdes, mais un certain nombre étaient en dégénérescence et leurs flagelles avaient disparu. 
Quelques spermatozoïdes étaient également visibles, au milieu des œufs, dans le canal utérin proche du pore génital (fig. 50).

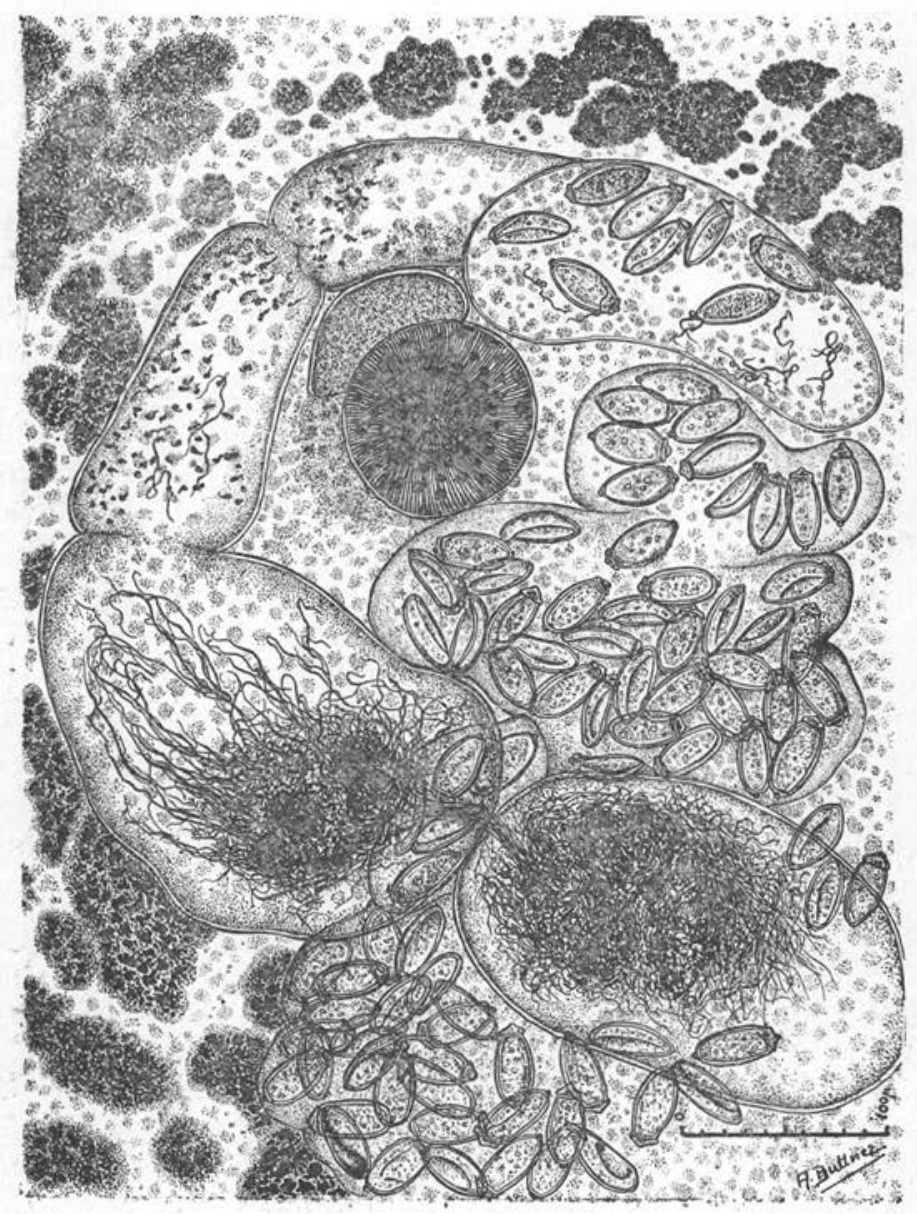

Fig. 50. - Partie $a \longleftrightarrow-b$ de ja figure 51, montrant le double renflement de la vésicule séminale contenant les amas agglutinés de spermatozoïdes. Remarquer les petites têtes de spermatozoïdes atypiques dans le manchon prostatique et la présence de quelques spermatozoïdes normaux parmi les œufs dans la partie terminale de l'utérus. (Orig.).

Les deux autres trématodes ne présentaient chacun qu'un seul amas de spermatozoïdes dans le renfflement de la vésicule séminale le plus éloigné de l'acetabulum ; la portion terminale de l'appareil 
génital mâle était dépourvue de spermatozoïdes, de même que les dernières anses utérines. Chez ces deux distomes, l'agglutinat formait une masse plus compacte et on ne pouvait voir de spermatozoïdes nettement isolés; la dégénérescence semblait certaine.

Chez ces trois trématodes, les deux testicules conservaient les mêmes caractères de dégénérescence que chez les métacercaires progénétiques de discoglosses, provenant de la collection $\mathrm{E}$. Brumpt : même structure vacuolaire, avec de petites cellules indifférenciées en division ; aucune figure typique de spermatogénèse.

Un second Zamenis, envoyé du Maroc par le $\mathrm{D}^{\mathrm{r}}$ Maurice, nous a servi de témoin. Après autopsie, il ne présentait que quelques nématodes dans la portion terminale de l'intestin, non loin du cloaque ; aucun trématode provenant d'infestations spontanées n'a été décelé. Rappelons que la biologie de cette couleuvre, qui vit dans les endroits secs, l'expose plus rarement sans doute que les couleuvres aquatiques des espèces Natrix natrix ou Natrix viperinus à des infestations naturelles par des trématodes de batraciens. Ch. Joyeux, qui en a examiné deux exemplaires venant du Jardin d'Essais d'Alger en 1929, n'a trouvé aucun trématode dans leur intestin, et R.-Ph. Dollfus (1929, p. 202) dit également qu'ayant disséqué plusieurs couleuvres fer à cheval à Rabat (Maroc), c'est dans l'intestin de l'une d'elles seulement qu'il a trouvé les trois exemplaires de Ratzia qu'il a décrits.

L'interprétation des résultats de nos expériences d'infestation de Zamenis hippocrepis, rapportés plus haut, nous laisse profondément perplexe.

Nous avions, en effet, examiné un assez grand nombre de métacercaires progénétiques de Ratzia joyeuxi de discoglosses (25 préparations), provenant de la collection E. Brumpt; aucune d'elles ne montrait, après coloration, de spermatozoïdes dans les testicules, ni dans le tractus génital, et la dégénérescence précoce des testicules nous a fait conclure en faveur d'une reproduction par parthénogénèse.

La présence indubitable de nombreux spermatozoïdes dans la vésicule séminale des trois trématodes, parfaitement vivants et actifs, retrouvés dans l'intestin grêle de Zamenis hippocrepis, peut être justifiée par différentes hypothèses :

$1^{\circ}$ Il s'agirait, non des Ratzia joyeuxi ingérées expérimentalement, mais de trématodes préexistant spontanément, comme c'était vraisemblablement le cas pour les trois Ratzia observées par R.-Ph. Dollfus au Maroc, en 1929, et que nous avons désignées sous le nom de Ratzia dollfusi (=R. parva Dollfus 1929, non Stossich 1904). 
$2^{\circ}$ Les trois distomes observés seraient bien trois Ratzia joyeuxi, subsistant parmi les 17 métacercaires progénétiques ingérées expérimentalement; le milieu intestinal de l'hôte aurait déclenché chez ces helminthes une spermatogénèse active et fugace, aussitôt suivie par une dégénérescence des testicules. Les agglutinats présents dans la vésicule séminale seraient des amas résiduels de spermatozoïdes, également en voie de dégénérescence, témoins de cette fugitive activité génitale. L'existence de spermatozoïdès dans l'utérus. près du pore génital, indiquerait que les œufs sont le produit probable d'une autofécondation. Il faudrait envisager dans ce cas la possibilité de deux processus de reproduction, parthénogénèse ou autofécondation, selon que la maturation du distome se réalise chez Discoglossus pictus ou chez Zamenis hippocrepis.

$3^{\circ}$ Il s'agirait de trois Ratzia joyeuxi, provenant des métacercaires ingérées lors de l'expérience la plus récente ( ${ }^{\circ}$ 688/A.B.-I); mais ces trématodes appartiendraient à une variété particulière de Ratzia joyeuxi, chez laquelle la spermatogénèse s'accomplirait déjà au stade métacercaire chez le discoglosse, les œufs progénétiques étant alors un produit normal d'autofécondation. S'il en était ainsi, le séjour dans le tube digestif de Zamenis hippocrepis aurait pour seul effet de digérer le kyste ; mais le vieillissement du distome et sa sénilité sexuelle s'y accompliraient dans les mêmes conditions que dans l'habitat sous-cutané qu'il occupe chez le discoglosse.

Il nous eût été bien facile de vérifier cette dernière hypothèse si nous avions pu prévoir les résultats inattendus de cette expérience. Quelques préparations colorées de métacercaires de discoglosses nous auraient permis de savoir si, chez ce batracien, l'activité spermatogénétique se manifestait déjà. Mais, comme nous l'avons signalé plus haut, nous ne possédions que peu de kystes vivants, tandis que nous avions de nombreuses préparations fixées en 1920 par le Professeur E. Brumpt.

Nous croyons pouvoir écarter tout de suite la première hypothèse : en effet, l'étude morphologique des trois distomes trouvés par nous chez le Zamenis permet de conclure avec une quasi-certitude à leur homologation aux métacercaires progénétiques de Ratzia joyeuxi.

Nous donnons ici une figure de l'un de ces trématodes (fig. 51) que l'on pourra comparer avec celle de Ratzia joyeuxi du discoglosse (fig. 46), bien que cette dernière soit un peu schématisée : la partie antérieure présente une cuticule spinulée, facilement observable même après coloration chez les trois trématodes ; la spinulation s'arrête un peu au-dessus du plan médian du corps (fig. 51, ar. $s p$.) comme chez $R$. joyeuxi; la vessie (V) est volumineuse 


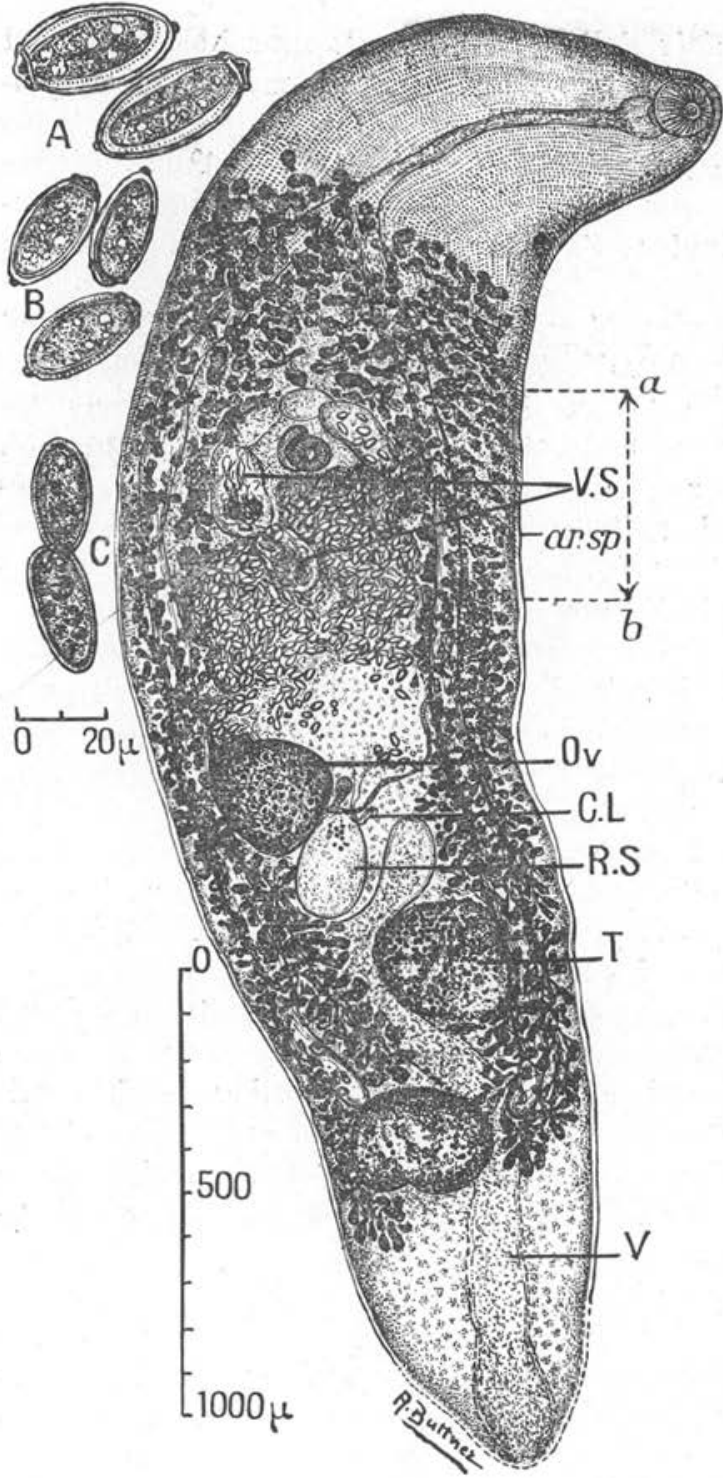

FIG. 51. - Ratzia joyeuxi var. inexpectata, A. Buttner, 1951. Exemplaire ingéré par Zamenis hippocrepis, trouvé dans l'intestin grêle. $-a \longleftrightarrow-n \rightarrow b$, partic reproduite au fort grossissement (fig. 50); ar.sp.: arrêt de la spinulation ; C.L. : canal de Laurer ; Ov. : ovaire ; R.S. : receptaculum seminis; T. : testicule ; V. : Vessie ; V.S. : double renflement de la vésicule séminale renfermant des spermatozoïdes agglutinés, - A, B, C, œufs de taille décroissante depuis le pore génital jusqu'à la sortie de l'ovaire. (Orig.). comme chez cette métacercaire et les gland e s génitales sont également en dégénérescence et n'ont pas atteint un volume supérieur à celui présenté par les Ratzia joyeuxi, au même degré de développement, que nous avons examinées ; le réceptacle séminal est sensiblement moins important que l'ovaire et ne contient pas d'éléments germinaux.

Chez $R$. dollfusi, rappelons que la cuticule n'est pas spinulée et ne présente qu'une fine ponctuation; la vessie est étroitement tubuliforme; les testicules sont très développés, parfaitement homogènes e $t$ fonctionnels; le réceptacle séminal est largement plus gros que l'ovaire et rempli, comme la vésicule séminale et le canal déférent terminal, de très nombreux spermatozoïdes.

Dans ces conditions, il nous semble que, morphologiquement et physiologiquement, les Ratzia observées dns l'intestin de notre Zamenis 
sont différentes de Ratzia dollfusi, tandis que morphologiquement elles apparaissent en tous points semblables aux métacercaires progénétiques de $R$. joyeuxi.

La seconde hypothèse, selon laquelle une spermatogénèse intense aurait été déclenchée sous l'effet de facteurs biologiques appartenant au milieu intestinal de Zamenis hippocrepis, nous retiendra davantage.

Observons tout d'abord qu'il peut paraître singulier, si l'on admet que la couleuvre fer à cheval est vraiment l'hôte chez lequel s'accomplit le terme de l'évolution de Ratzia joyeuxi, que sur les 17 métacercaires ingérées, trois distomes seulement aient subsisté. Il est vrai que nous avons pu constater (1) combien le pouvoir infectieux d'autres espèces de métacercaires était sujet à variations. Il se pourrait que, chez cette espèce de trématode, le pourcentage d'évolution soit faible chez l'hôte définitif normal.

Un second point à envisager est le degré avancé d'évolution des distomes ingérés qui, chez le discoglosse, sont déjà sexuellement déterminés, puisqu'ils pondent des œufs en abondance et font parfois éclater le kyste, répandant les œufs dans les tissus environnants. Il faudrait donc admettre que les testicules ne peuvent devenir fonctionnels chez le discoglosse, en raison peut-être d'une carence hormonale, tandis que, chez le Zamenis, ce facteur biologique serait présent et provoquerait le développement et le fonctionnement des glandes génitales mâles.

Cependant, même dans cette éventualité, il paraît surprenant qu'en 8 ou 40 jours (durées de séjour dans l'intestin des métacercaires absorbées), puissent se dérouler une succession de phénomènes tels que la normalisation des testicules, leur entrée en activité et surtout leur dégénérescence les restituant exactement, comme chez nos trois exemplaires, dans l'état histologique où ils se présentent habituellement chez le discoglosse.

Ces processus supposent des modifications cytologiques profondes qui s'observent en général chez des individus jeunes. Cependant, Ch. Joyeux, qui avait fait ingérer des kystes de tous âges à des couleuvres des genres Tropidonotus (aquatique) et Coluber (terrestre), n'a retrouvé, au bout de 14 jours, qu'un seul exemplaire de Ratzia joyeuxi chez une Tropidonotus natrix; les testicules étaient en pleine dégénérescence et Joyeux ne signale pas avoir observé de spermatozoïdes dans l'appareil génital mâle.

Dans cette seconde hypothèse, il faudrait donc supposer qu'un facteur biologique particulier, existant spécifiquement chez Zame-

(1) Brumpt (E.) et Buttner (A.), Ann, Paras., 1949. 
nis hippocrepis, permettrait seul l'évolution normale des testicules de $R$. joyeuxi chez cette couleuvre, à l'exclusion d'autres colubridés communs, terrestres ou aquatiques. Cette action ne s'exercerait sans doute que sur des métacercaires jeunes ou de maturité récente, ce qui rendrait compte du faible pourcentage d'évolution des kystes ingérés.

Toutefois, la spécificité étroite de cette métacercaire pour un hôte définitif qui semble rarement spontanément infesté, son faible pouvoir infectieux expliquent difficilement la pérennité de ce parasitisme dans la nature; à moins qu'un cycle progénétique, réalisé par parthénogénèse, n'existe concurremment pour assurer la conservation de l'espèce.

Bien qu'il ne nous soit pas possible d'en donner une preuve expérimentale, nous préférons nous rallier à la troisième hypothèse qui nous semble plus proche d'un processus naturel : une spermatogénèse normale, active, se développant pendant la maturation sexuelle de certaines métacercaires et atteignant son plein effet lors de la progénèse, suivie de la dégénérescence des testicules et de la persistance pendant quelque temps d'une accumulation de germes inemployés dans les poches séminales, justifierait très simplement les anomalies sexuelles des distomes récoltés chez notre Zamenis. Nous nous trouverions, dans ce cas, en présence d'une variété biologique de Ratzia joyeuxi. Le problème reste pendant. En attendant que de nouvelles expériences lui apportent peut-être une solution, et en raison du comportement inattendu du distome dont nous venons d'étudier l'évolution, nous le désignons sous le nom de Ratzia joyeuxi inexpectata nov. var.

\section{Etude de la reproduction}

\section{A. Parthénogénèse}

Bien qu'il ne ressorte pas clairement des expériences de Ch. Joyeux si les œufs de métacercaires qu'il a étudiẹs provenaient de $R$. joyeuxi de Discoglossus ou de R. parva de Rana, bien qu'il ait observé tantôt leur maturation en 15 jours, tantôt leur éclosion au bout d'une heure dans le milieu extérieur, nous admettrons que les œufs pondus dans les kystes mûrs de $R$. joyeuxi sont embryonnés. Nous avons en effet eu l'occasion de voir un miracidium bien formé dans ces œufs, observation déjà consignée par E. Brumpt dans ses protocoles expérimentaux inédits.

Ici, se pose donc à nouveau le problème du processus de la reproduction dans un autokyste, où le distome se trouve enfermé, éliminant, par conséquent, toute possibilité de fécondation croisée. 
Nous avons examiné un certain nombre de préparations in toto, fixées et colorées (environ 25), ainsi que des coupes de kystes de Ratzia joyeuxi (Brumpt-1922), prélevés chez des discoglosses en 1920, par E. Brumpt.

Déjà, chez les exemplaires jeunes $(2 \mathrm{~mm}$. de long), où la production d'œufs en est encore à ses débuts, les testicules présentent un commencement de dégénérescence, une structure vacuolaire et une activité cellulaire qui ne semble pas aller au delà du stade de multiplication.

Les exemplaires plus âgés (4-5 mm.) montrent cependant des testicules plus gros, quoique de plus en plus dégénérés; les cellules sont anormales, dissociées. Le tractus génital mâle s'est néanmoins complètement développé et on distingue nettement les canaux déférents, la vésicule séminale bi- ou tripartite, enfin son manchon terminal. L'ovaire, qui ne s'efface que plus tard, à la fin de l'activité génitale, possède un réceptacle séminal aussi volumineux que lui.

L'activité testiculaire paraît ainsi déviée et, à aucun moment, il ne nous a été possible d'observer la spermatogénèse, dont les stades caractéristiques se reconnaissent si facilement. Il semble qu'ici, les glandes génitales mâles aient perdu la faculté de différenciation de leurs éléments germinaux en spermatides et spermatozoïdes fonctionnels, et que leur action se borne à la production des spermatogonies. Le receptaculum seminis est ainsi partiellement rempli de cellules en division et de produits de sécrétion anormaux. Aucun spermatozoïde n'est visible dans les anses utérines. La vésicule séminale, quoique bien développée, reste vide, tandis que, dans les exemplaires adultes de l'intestin de Zamenis, les spermatozoïdes foisonnent dans l'appareil génital mâle jusqu'à l'orifice terminal.

Par quelle fantaisie de la nature, ce distome, chez lequel l'orifice utérin est perméable, puisque de nombreux œufs sont évacués dans le kyste, et où rien ne s'oppose donc à une autofécondation, semble-t-il effectuer son évolution par parthénogénèse ?

Chez les exemplaires de Ratzia parva de Rana esculenta, on observe la même inhibition testiculaire : aucune figure typique de spermatogénèse ; les glandes génitales restent petites, mais les testicules paraissent dégénérer moins rapidement que chez $R$. joyeuxi.

Cependant, le développement des œufs se produit normalement chez ces deux espèces progénétiques et l'on peut suivre l'évolution de la cellule ovulaire jusqu'au miracidium. La fécondité est très grande et des centaines d'œufs ont été produits lors de la déhiscence du kyste. Toutefois, l'ovaire ne grossit pas sensiblement et son volume est généralement inférieur à celui des testicules. Les œufs évacués dans le kyste sont fréquemment ingérés par le distome 
qui tourne dans son kyste. Ce phénomène s'observe d'ailleurs plus souvent chez $R$. parva que chez $R$. joyeuxi. Notons que, dans ce cas, le milieu intestinal de ces Ratzia ne suffit pas à faire éclore les œufs.

\section{B. Fécondation}

Faisant état de notre récente expérience sur Zamenis hippocrepis (L.), il nous faut envisager ici la possibilité pour Ratzia joyeuxi de se reproduire également par une fécondation normale.

En effet, si la variété inexpectata existe réellement, il y a peutêtrè déjà, au stade métacercaire, autofécondation, celle-ci ne faisant que se poursuivre, après ingestion, chez Zamenis hippocrepis.

$\mathrm{Si}$, au contraire, cette variété s'intègre dans une espèce unique, le processus normal de la fécondation succéderait à la parthénogénèse, en raison du changement d'habitat de la métacercaire. Les deux processus existeraient donc en puissance chez cette dernière, mais ne se réaliseraient que sous l'effet de facteurs biologiques appartenant à l'un ou l'autre de ses hôtes.

Quoi qu'il en soit, ce distome peut se multiplier par une fécondation normale, et c'est la présence de ce double mécanisme de reproduction chez des individus mûrs appartenant à une même espèce qu'il nous a paru intéressant de souligner ici.

\section{Conclusions}

- Le cas de Ratzia joyeuxi (Brumpt 1922), second exemple de progénèse constante, obligatoire, semble, comme le précédent (Paralepoderma brumpti), sous la dépendance étroite de facteurs héréditaires.

-- Aucune influence extérieure ne paraît d̀ priori capable de modifier son développement chez le discoglosse. Sans doute, la saison intervient-elle pour favoriser plus ou moins l'évolution de ce distome. Toutefois, nous savons qu'à une époque tardive (octobre), Joyeux a encore trouvé 7 discoglosses infestés sur 11 récoltés.

- Y a-t-il une action de l'hôte ?

Celle-ci demeure incertaine, car elle suppose la résolution de problèmes préliminaires, non encore élucidés.

Etant donné leur spécificité parasitaire différente, nous avons jugé préférable, tout au moins provisoirement, de faire deux espèces distinctes de la métacercaire progénétique de Rana esculenta, enkystée dans les muscles [= Ratzia parva (Stoss.)], et de celle de Discoglossus pictus, enkystée sous la peau $[=$ Ratzia joyeuxi 
(Brumpt)]. Mais cette non-identité reste à démontrer expérimentalcment.

Pour cela, il faudrait qu'à partir de mollusques neufs, parasités au laboratoire avec les œufs embryonnés d'un individu mûr (métacercaire progénétique ou adulte vrai), on expose simultanément à l'attaque des cercaires émises par ces mollusques des Discoglossus pictus et des Rana esculenta. La réceptivité ou non-réceptivité de l'une ou l'autre de ces espèces de batraciens, d'une part, la localisation des kystes en surface ou en profondeur, soit chez les deux espèces, soit spécifiquement chez chacune d'elles, d'autre part, permettraient de se prononcer sur leur identité véritable.

S'il n'y a en fait qu'une seule espèce de Ratzia, l'action de l'hôte pourrait rendre compte des légères variations morphologiques qui séparent les distomes du discoglosse de ceux de la grenouille, et de leur localisation distincte. S’il s'agit d'espèces différentes, le problème ne se pose plus sous cet angle.

Les expériences d'évolution chez Zamenis hippocrepis soulèvent la question de savoir s'il y a véritablement eu « évolution » chez ce colubridé et si cet hôte est réellement intervenu pour modifier la physiologie sexuelle de la métacercaire progénétique.

Ce problème ne pourra être résolu sans de nouvelles expériences. portant sur plusieurs couleuvres fer à cheval, avec un nombre important de kystes à divers degrés de maturité.

Il faudrait, en outre, que de nombreux kystes, prélevés parmi ceux que l'on ferait absorber aux Zamenis, fussent fixés, colorés et soigneusement examinés, afin de rechercher s'il ne se trouve pas quelques métacercaires présentant déjà à ce stade une activité testiculaire. Une semblable observation confirmerait la création d'une variété particulière (inexpectata, nov. var.) de Ratzia joyeuxi ; mais il resterait à trouver si le passage par un hôte définitif devient de ce fait nécessaire à l'achèvement de sa maturation ou si le rôle de cet hôte se borne à une tolérance passive, purement accidentelle.

Observons, à ce propos, que le nombre de kystes progénétiques ayant fait l'objet d'examens sur préparations par E. Brumpt, Ch. Joyeux, ainsi que par R.-Ph. Dollfus avec le matériel de ces auteurs, ne dépasse probablement pas 50, chiffre insuffisant pour donner une idée statistique des caractères sexuels de ces métacercaires. Ces auteurs ne signalent pas avoir observé de spermatogénèse ; nous-même n'en avons jamais décelé après une étude attentive de 25 préparations colorées de la collection E. Brumpt. Néanmoins, il est peut-être aventureux de généraliser trop vite ces observations à toutes les métacercaires de Ratzia joyeuxi.

$\mathrm{Si}$, cependant, une statistique portant sur un nombre élevé d'in- 
dividus permettait de conclure que les métacercaires progénétiques sont parthénogénétiques, et si leur évolution répétée chez des Zamenis faisait apparaître, de façon constante, un réveil de leur activité spermatogénétique, force nous serait de reconnaître une action qualitative de l'hôte sur la physiologie sexuelle du distome. Mais, nous l'avons discuté plus haut, cette éventualité nous parait douteuse.

Toute l'évolution de Ratzia joyeuxi reste essentiellement originale et elle pose à notre sagacité des problèmes inhabituels chez les trématodes :

$1^{\circ}$ En ce qui concerne l'affinité des cercaires pour un hôte généralement peu sensible à leurs attaques. Il n'y a presque pas d'exemple (1), à notre connaissance, de cercaires batracophiles qui, négligeant les têtards, lesquels existent néanmoins au printemps dans les mêmes gîtes, montrent une préférence pour l'hôte vertébré adulte. Cette prédilection est à ce point marquée, que certains discoglosses sont parfois entièrement recouverts de kystes (fig. 8). Sous l'effet de quel tropisme cette attraction s'exerce-t-elle ? Nous ne pouvons actuellement que constater ce fait comme un comportement particulier de ce distome.

$2^{\circ}$ En ce qui concerne le cycle évolutif de Ratzia joyeuxi qui, selon toute vraisemblance, est un cycle abrégé, comportant peut-être des modalités de reproduction chez la métacercaire.

Comment, en effet, expliquer la persistance, au Jardin d'Essais d'Alger, depuis au moins 20 ans, de ces formes progénétiques, non pas accidentelles, mais constantes, si la majeure partie d'entre elles ne jouait aucun rôle dans la conservation de l'espèce?

Il est possible même, étant donné que ce parasitisme est fréquent dans la nature, que le cycle abrégé se réalise facilement et que les échecs expérimentaux enregistrés jusqu’à présent proviennent de ce que le prosobranche vecteur n'a pas encore été trouvé, la spécificité pour le premier hôte intermédiaire étant en général, d'après nos expériences sur les trématodes digénétiques, extrêmement étroite.

$3^{\circ}$ En ce qui concerne le processus de reproduction de cette espèce. La parthénogénèse apparaît, dans ce cas, comme un phénomène gratuit, difficilement justifiable. Comme nous le faisions

(1) Rappelons que Leigh et van Cleare (voir Ann. Paras. XXVI, 1951, p. 43, note 1), à propos de l'afinnité particulière des cercaires de Glypthelmins quieta pour des Rana adultes et de leur répulsion pour les têtards de cette espèce encore privés de pattes, ont montré que l'administration à ces têtards d'extrait thyroïdien accélérant la métamorphose suffisait à déterminer l'enkystement des cercaires. Il y a là un histotropisme particulier résultant peut-être de l'entrée en activité de la séerétion thyroïdienne. 
observer à propos de l'étude de la fécondation des œufs chez Paralepoderma brumpti, il est de fait que tout organe existant en puissance chez l'embryon ne subsiste qu'à l'état rudimentaire chez l'adulte, s'il n'est pas appelé à jouer un rôle physiologique. Or, chez Ratzia joyeuxi, nous nous trouvons en présence d'un appareil génital mâle entièrement développé ; les testicules quoiqu'anormaux grossissent au cours du développement, et bien que n'étant pas fonetionnels, ils manifestent une certaine activité mitotique; les cellules en division semblent même mobiles, puisquę nous les retrouvons parfois dans le réceptacle séminal dépendant du tractus ovarien.

Nous pensons que ce phénomène est un argument de plus en faveur du caractère strictement génétique de la progénèse dans ce cas particulier. Elle ne semble pas résulter d'une adaptation progressive à des conditions favorables au développement progénétique, mais d'une mutation brusque, venue troubler soudain l'équilibre physiologique du distome. L'appareil génital mâle persisterait comme un témoin de son ancien état fonctionnel.

Toutefois, la présence de spermatozoïdes dans le tractus génital des Ratzia, retrouvés par nous dans l'intestin grêle de Zamenis hippocrepis (exp. 677/A.B.-I), nous oblige à reconnaitre que ces distomes sont également aptes à se reproduire par une fécondation normale. S'agit-il d'une variété particulière ? Cette variété, en admettant qu'elle existe, est-elle rare, est-elle aussi progénétique ? Ou bien a-t-elle échappé à la mutation présumée, et représente-t-elle une survivance d'un cycle antérieur à trois hôtes ?

Nous ne prendrons pas position, car ce sont là autant d'énigmes qui ne trouveront leur solution que par l'expérimentation.

$4^{\circ}$ En ce qui concerne la croissance et la maturation du distome dans un autokyste. Nous avons déjà évoqué ce problème dans le chapitre précédent. Nous ne faisons que le rappeler pour signaler à nouveau cette curieuse adaptation à un genre de vie très spécial. Signalons qu'ici, encore, le kyste est mince et laisse sans doute facilement passer par osmose les substances nutritives nécessaires à la croissance et à la différenciation de l'animal. Sa paroi est extensible : l'animal se développe à l'intérieur du kyste qui s'agrandit progressivement. Il serait intéressant de connaître la nature des substances filtrables dont se nourrit le trématode enkysté, dans cet habitat superficiel. Nous n'avons pas abordé ce problème; il est de fait que ce distome est le siège d'un métabolisme très actif, car la vessie est toujours très volumineuse et remplie de produits d'excrétion. Ceci nous engage à conclure que la vie enkystée n'est pas une cause de ralentissement du développement 
chez les formes progénétiques : nous avons vu en effet cette évolution se produire en moins de 15 jours chez Paralepoderma brumpti, enkysté dans les muscles des têtards; l'activité physiologique de Ratzia joyeuxi, dans son kyste, nous paraît être le témoignage d'une évolution s'effectuant normalement, dans des conditions favorables.

\section{Pleurogenes medians (Olsson, 1876)}

Avec ce troisième cas de progénèse, nous abordons une forme de développement précoce assez différente de celle manifestée par les deux distomes précédemment étudiés.

\section{Premières observations expérimentales. Description du distome}

La première métacercaire progénétique de Pleurogenes medians a été signalée par Sinitzin en 1905, dans sa thèse publiée en russe (1) ; mais, dans le résumé français qu'il en donne, en 1907, cet auteur se contente de dire, à propos de Distomum medians Olsson, qu'il a trouvé des kystes avec les formes jeunes de ce parasite dans le corps des agrions et des larves de petits coléoptères.

Après Sinitzin, P. Mathias (1924), récoltant en février et avril des Gammarus pulex dans un petit ruisseau du Bois de Boulogne, voisin de la Porte-Maillot, vit, soit dans leur corps, soit dans les articles des pattes ou des antennes, une moyenne de 8 à 12 et parfois jusqu'à 25 métacercaires sphériques, mesurant de 350 à $610 \mu$ de diamètre, la taille la plus habituelle étant de $410 \mu$.

Ces kystes à paroi peu épaisse éclataient facilement par simple écrasement; le trématode ainsi libéré mesurait 610 à $820 \mu$ de long sur 350 à $450 \mu$ de large. Son corps était entièrement couvert d'épines ; la vessie, remplie de produits d'excrétion, était en forme de V et se détachait en noir ; le tube digestif comportait un pharynx. musculeux, un œsophage et deux cæcums courts atteignant à peine le bord antérieur de la ventouse ventrale. Les deux testicules étaient symétriques, de part et d'autre de l'acetabulum, et une volumineuse poche du cirre s'ouvrait non loin de la ventouse orale, sur le côté droit de l'animal vu par sa face ventrale, tandis que l'ovaire, à gauche, se trouvait entre le bord externe du cæcum et la cuticule. Les glandes vitellogènes se développaient à la partie antérieure du distome, juste au-dessus de la ventouse ventrale.

(1) Pl. IV, fig. 68 : Métacercaire de Pleurogenes medians dékystée mais non progénétique. 
Mathias avait été frappé par la ressemblance de ces métacercaires de gammares avec le ver adulte de Pleurogenes medians (Olsson), parasite de l'intestin antérieur et moyen de diverses espèces de batraciens. Il fit ingérer un certain nombre de kystes de grande taille, mais non progénétiques, à des Rana temporaria indemnes de trématodes (aucun œuf de ces helminthes n'ayant été trouvé dans leurs déjections), et obtint en 12 jours, dans leur intestin grêle, des distomes présentant de nombreux œufs mûrs dans leur utérus, incontestablement identifiables à Pleurogenes medians (Olss.). Il répéta cette expérience avec les mêmes résultats chez une Hyla arborea L.

Ce n'est qu'en juin 1924 que l'auteur constata, chez de nouveaux gammares récoltés dans le même gìte, la présence de métacercaires progénétiques qui, sorties de leur kyste, semblaient de véritables adultes de Pleurogenes medians (Olss.).

C'était la première fois que des kystes progénétiques de Pleurogenes medians avaient été observés chez des gammares. Ce fait a ćté signalé à la même époque par R.-Ph. Dollfus pour des métacercaires progénétiques de gammares que lui avait confiées le Professeur E. Brumpt. Dollfus fait remarquer que cette polyxénie de la métacercaire étend le nombre des combinaisons d'hôtes que l'on peut prévoir pour le cycle évolutif de l'espèce.

Les métacercaires enkystées spontanément chez Gammarus pulex L. sont nombreuses, Mathias en signale trois espèces (fide Lühe) : D. gammari Linstow 1877 (kyste sphérique), D. pulicis Linst. 1892 et Dist. species La Val. 1859 (kystes ovales). Dollfus rappelle, en outre, celles de von Siebold (1848), d'Engel (1865) qui rapporte que, faisant ingérer ces métacercaires à des grenouilles, il obtint des adultes identifiés par lui à Opisthioglyphe endolobum Duj., d'A.P. W. Thomas (1881), et une observation de métacercaire enkystée chez Gammarus pungens Edw. en Tunisie, par Pr. Sonsino (1893-1894). Signalons encore le Distoma agamos v. Linst. (1872), enkysté chez Gammarus pulex L., chez lequel il y a production d'œufs et de spermatozoïdes, et dont la forme adulte est identifiée par Lühe à Allocreadium transversale Rud.

Enfin, nous avons nous-même constaté que les gammares étaient très sensibles aux attaques des cercaires. A Richelieu, nous avons obtenu facilement l'enkystement expérimental chez cet amphipode de plusieurs xiphidiocercaires entomophiles, en particulier de la cercaire décrite par E. Brumpt en 1944-1945, Xiphidiocercaria polyxena (expérience 147/A.B.-I). Dans la nature, sur 159 gammares examinés, provenant de la rivière Mable (expérience 79/I.A.B.), nous en avons trouvé près de 100 présentant des kystes de toute taille, souvent grégaires et où le stylet était parfois présent. Deux 
ont présenté une métacercaire de grande taille, avec une vessie excrétrice simple et très grande rappelant celle appartenant à la famille des Allocreadiidæ (expérience 127/A.B.-I). Dans ce gîte, nous n'avons jamais observé de métacercaires progénétiques chez les gammares entre mai et septembre, au cours des étés 1948 et 1949. Il y a quelques années, nous avons observé, avec le Professeur E. Brumpt, chez un Echinogammarus berilloni, originaire de Thuet (Indre-et-Loire), des métacercaires progénétiques de Coitocæcum sp. (fase. 4, fig. 59).

\section{Etude de l'évolution. Comment se manifeste la progénèse chez} PI. medians d'après les auteurs. - Rappelons que le cycle évolutif type des espèces de la sous-famille des Pleurogenetinæ a été découvert par Okabe (1937), pour Pleurogenes liberum (Seno 1907), dont le genre Loxogenes n'est qu'un synonyme aujourd'hui abandonné. Cet auteur a constaté qu'une cercaire virgule, émise par Bithynia kiushuensis et Bithynia striatula japonica, s'enkystait chez des Odonates (Crocothemis servilia) et que l'adulte se rencontrait chez différents batraciens du Japon. Okabe n'a pas signalé de métacercaires progénétiques. Cette même espèce a été étudiée par Yamaguti (1938).

Yamaguti a vu que ces cercaires virgules, comme toutes celles de ce groupe, naissaient dans de petits sporocystes observés chez B. striatula, var. japonica. Il a obtenu facilement des infestations expérimentales chez deux espèces de libellules : Crocothemis servilia et Orthetrum albistylum. L'adulte se développe chez des batraciens, en particulier des grenouilles.

L'évolution partielle d'une autre espèce de Pleurogenes, $P$. arcanum (Nickerson) a été obtenue par W. W. Crawford en 1938. Cet auteur n'a pas décrit la cercaire ; à partir des métacercaires trouvées chez plusieurs espèces de libellules (9), il a infesté Rana pipiens et Rana clamitans. Ce trématode se rencontre dans la nature chez plusieurs espèces de batraciens. Bien qu'il ait eu affaire à des infestations naturelles datant probablement de plusieurs mois, Crawford ne signale pas de métacercaires progénétiques. Signalons toutefois la curieuse biologie de ce ver qui, par son habitat, peut être rapproché de Brandesia parva. En effet, les vers sortant des métacercaires restent libres six à sept jours dans le duodénum, puis s'enkystent isolément ou parfois par groupes de deux, trois ou quatre, dans la paroi du duodenum. Les adultes sont fertiles au bout de quatre à cinq semaines et atteignent leur croissance totale en huit à dix semaines. L'auteur ne dit pas toutefois comment les œufs sont libérés dans le milieu extérieur. 
P. Mathias, en 1924, a apporté une intéressante contribution à l'étude du cycle évolutif de Pleurogenes medians en faisant ingérer des métacercaires non progénétiques à des batraciens anoures, ce qui lui a permis d'en faire la détermination certaine et d'établir la durée de leur évolution en adultes, soit douze jours.

Ch. Joyeux, R. du Noyer et J.-G. Baer (1930) ont à leur tour étudié l'évolution de ce distome. Toutefois, une interprétation erronée d'une observation de Mathias à la fin de sa note, dans laquelle cet auteur mentionne que les seuls mollusques présents dans le gîte sont des Limnæa limosa et des Bithynia tentaculata, concluant de ce fait que la cercaire de Pleurogenes medians ne doit se développer que chez l'un de ces mollusques, les a mal orientés.

Joyeux, du Noyer et Baer disent simplement que "Mathias a soupçonné la cercaire d'être hébergée par Limnæa limosa»; or, ce mollusque est un pulmoné et l'on sait que, obéissant à une loi biologique à peu près constante, les espèces d'une même famille de trématodes évoluent toujours chez la même catégorie de mollusques, soit pulmonés, soit prosobranches. Etant donné les observations d'Okabe et de Yamaguti concernant le cycle évolutif de Pl. liberum chez des Bithynia émettant des cercaires virgules, il était peu vraisemblable que $\mathrm{Pl}$. medians fìt son évolution chez des mollusques pulmonés.

Toutefois, reprenant les recherches de Mathias dans le même gîte, les trois auteurs ont trouvé une xiphidiocercaire très active chez Limnæa limosa, tandis que « des Planorbis corneus et des Bithynia tentaculata étaient indemnes ».

Nous ne signalerons donc pas les expériences d'infestations expérimentales de ces auteurs qui, si elles présentent un intérêt biologique pour les xiphidiocercairies, ne peuvent apporter de contributions nouvelles à l'étude dv *vele évolutif de Pleurogenes medians depuis que l'on sait, par le travail de Neuhaus (1940 et 1941), que sa cercaire est une cercaire virgule, émise par Bithynia tentaculata.

Nous ne mentionnerons que les observations effectuées par Joyeux, Rondeau du Noyer et Baer (1930), à différentes époques de l'année sur l'infestation naturelle des gammares du gîte du Bois de Boulogne; ceci, afin de pouvoir les comparer à celles que nous avons faites récemment dans les mêmes conditions, mais sur un hôte intermédiaire nouveau, la larve de Sialis lutaria.

Ces observations des auteurs précités ont fait l'objet de deux séries de recherches.

Dans la première série, les trois auteurs ont donné le résultat d'examens périodiques pratiqués pendant une année sur les gam- 
mares du Bois de Boulogne, dans le but de suivre l'évolution génitale de la métacercaire :

- 12 juillet 1929 : présence de kystes de $125 \mu$ de diamètre, mais pas d'organes formés.

- 18 septembre 1929: deux sortes de kystes (300 et $150 \mu$ de diamètre) de la même espèce (Pleurogenes medians); les plus petits proviendraient d'une deuxième infestation, datant probablement du mois d'août. varié.

- 28 octobre 1929 : même aspect, les tailles respectives ont peu

- 24 décembre : les deux sortes de kystes sont difficiles à distinguer, la taille étant à peu près la même, soit environ $300 \mu$; testicules, ovaire, poche du cirre formés.

- 10 février 1930 : organes génitaux plus développés, aucune trace de progénèse ; 26 examens.

- 14 mars 1930 : organes génitaux très développés; en coupe, testicules montrant une spermatogénèse active; vésicule séminale bourrée de spermatozoïdes; cirre évaginé ; sur 44 métacercaires examinées, aucune progénétique.

- 9 avril 1930 : sur 32 métacercaires, 8 sont progénétiques; le nombre d'œufs dans l'utérus est de 12 à 15 , souvent un seul, à coque à peine formée. Ces œufs, présents dans la partie moyenne de l'utérus, ne paraissent pas se rapprocher de l'orifice de ponte. La cellule ovulaire est visible, ainsi que sa segmentation en blastomères ; les auteurs ajoutent que les métacercaires non progénétiques montrent également une grande activité génitale : "Chez toutes, la fécondation s'accomplit. 》

- 12 mai : sur 36 métacercaires, 26 progénétiques. Les kystes les plus gros sont généralement ceux chez lesquels la progénèse est la plus accentuée ; néanmoins, il peut y avoir des exceptions. Le même gammare peut héberger des métacercaires à divers degrés d'évolution ; en coupes, les organes génitaux apparaissent en pleine activité ; l'œuf se développe normalement; cependant un certain nombre sont dégénérés. Leur coque est froissée et ils ne contiennent qu'une substance amorphe.

- 8 juin : même aspect des métacercaires progénétiques; les œufs ne sont jamais très nombreux dans l'utérus : 20-30 au maximum; ils ne s'acheminent pas vers le pore utérin, et on n'en observe aucun à l'intérieur du kyste ; il n'y a donc pas de ponte. Dimensions des œufs $32 \times 15 \mu$ (Sinitzin donne $28 \times 18$, et R.-Ph. Dollfus $23-28 \times 18-19 \mu$ ).

- 11 juillet : "L'infestation de 1930 a commencé, et les gammares contiennent déjà de jeunes métacercaires à organes non 
développés d'infestation récente. On ne retrouve plus qu'un seul gammare hébergeant des métacercaires progénétiques de l'année précédente. Il est d'ailleurs également parasité par des métacercaires récentes; donc, pas de protection par une atteinte antérieure, comme nous l'avions déjà signalé ci-dessus. Les métacercaires progénétiques ne sont pas plus avancées que le mois précédent. Le nombre des œufs n'a pas augmenté; aucun phénomène de ponte. Les métacercaires semblent dégénérées.

Conclusion de ces auteurs : L'infestation des gammares a lieu en juin; les organes de la métacercaire ont évolué pendant l'hiver ; l'activité sexuelle est apparue au printemps, et la progenèse s'est manifestée peu après. Le nombre des œufs est toujours peu considérable, et leur constitution semblable à celle des œufs du trématode adulte ; la ponte à l'intérieur du kyste n'a jamais été observée. Les métacercaires semblent dégénérer environ un an après avoir pénétré chez l'hôte.

Dans une deuxième série d'observations, les auteurs ont étudié l'évolution de la métacercaire de Pleurogenes medians chez un grand nombre de gammares du Bois de Boulogne, transportés en juillet dans un aquarium du laboratoire de l'Evolution se trouvant dans une serre chauffée entre 18 et $25^{\circ}$ et alimenté par un courant d'eau. Bien que l'élevage ait été très florissant, les auteurs ont constaté que les métacercaires de ces gammares en captivité ont évolué tout différemment que dans la nature.

Des examens pratiqués pendant tout l'hiver ont établi que le développement des métacercaires était extrêmement lent :

- De juillet 1929 au 9 avril 1930 : les kystes ne mesuraient que $280 \mu$. Les glandes génitales sont en formation, la poche du cirre est à peine ébauchée, ainsi que le tube digestif. A cette époque, les auteurs observaient la progénèse chez des gammares en liberté.

- 12 mai : même état ; aucun progrès.

- 27 mai : quelques kystes atteignent $300 \mu$; l'évolution génitale n'a pas avancé.

- 16 juin : même état; les métacercaires semblent dégénérer.

Conclusion des auteurs: Pas de manifestation de progénèse, ni même fonctionnement des organes génitaux chez les métacercaires de $P l$. medians hébergées par des gammares en captivité. Cependant, ces amphipodes étaient placés dans des conditions particulièrement avantageuses, abondamment nourris, et ne semblaient pas souffrir.

L'évolution de la métacercaire, son activité génitale et la production d'œufs, seraient, d'après ces auteurs, "sous la dépendance des conditions dans lesquelles se trouve l'hôte qui l'héberge ». 


\section{Recherches personnelles}

Si nous relatons avec quelques détails les observations consignées par les trois précédents auteurs, c'est parce que, effectuant des recherches semblables, dans les mêmes gìtes, nous avons obtenu des résultats notablement différents.

Signalons d'abord qu'en raison de plusieurs années consécutives de sécheresse, le niveau des ruisseaux du Bois a considérablement baissé ; l'eau n'y est plus courante, et, au cours de nos prospections hebdomadaires, de décembre à mai, nous n'avons pour ainsi dire pas trouvé de gammares. En revanche, dans les fonds vaseux, les aselles étaient légions, et nous avons eu la bonne fortune de récolter fréquemment dans notre filet, parmi la boue et les feuilles mortes, des petites larves de teinte jaunâtre, avec des taches brunes sur la face dorsale du prothorax et de la tête, appartenant à l'espèce Sialis lutaria (insecte névroptère).

Cette larve, assez abondante en hiver, s'est révélée un matériel de choix pour l'étude de la progénèse. Elle supporte bien la captivité ; cependant, en raison de ses mœurs carnassières (elle est pourvue de deux fortes mandibules portant des dents sur le bord interne), on est obligé de l'isoler. L'abdomen est constitué par neuf segments, les sept premiers étant pourvus, de chaque côté, d'un appendice transparent trachéo-branchial, filiforme et cilié. Ces appendices présentent quatre étranglements, qui leur donnent un aspect curieux, pluriarticulé. Lorsque l'animal bouge, ils sont en général écartés du corps et relevés. Les pattes sont bien développées et terminées par deux griffes. Quand la larve se fixe, elle fait onduler son corps gracieusement pour mieux respirer. Cette larve marche, rampe, fouit, mais elle ne nage pas. Elle peut occasionnellement vivre plusieurs jours hors de l'eau et marcher sur le sol, même à reculons.

On n'est pas très fixé sur la durée de l'état larvaire (un an, peutêtre deux...). Cependant, dès le mois d'avril, cette larve devient plus difficile à trouver. C'est l'époque à laquelle elle sort de l'eau et va s'enfouir dans la terre ou la mousse pour se transformer en nymphe. L'imago est un névroptère, volant lourdement. Ses quatre aile: semblables ne peuvent se plisser. Il dépose ses œufs dans le voisinage de l'eau (contre les bateaux, sur les feuilles des végétaux rivevains, etc...) et il vit lui-même sur les pierres ou les bords des ruisseaux, non loin du gite aquatique qui a abrité sa forme larvaire. Nos prospections, suspendues en mai, ne nous ont pas permis de capturer des adultes, chez lesquels nous aurions voulu pouvoir étudier la destinée des métacercaires progénétiques hébergées par la 
larve. Nous pensons reprendre ces recherches lors de travaux ultérieurs sur la progénèse.

\section{Observation des infestations naturelles}

C'est à partir du 15 décembre que nous avons suivi, de huit jours en huit jours, l'évolution des métacercaires spontanées de Pleurogenes medians chez la larve de Sialis lutaria, pêchée dans le ruisseau du Bois de Boulogne aboutissant au pavillon d'Armenonville, après avoir contourné l'île des Cèdres et longé l'allée de Longchamp. Nos investigations ont bien entendu porté sur tous les ruisseaux alimentés par le lac supérieur, mais le gîte incontestablement le plus riche au point de vue du parasitisme par Pl. medians se situait dans le tronçon coulant entre l'île des Cèdres et l'allée de la Porte des Sablons à la Porte Dauphine.

Du 15 décembre au 28 février, nous avons disséqué environ 150 larves de Sialis ; celles-ci étaient infestées dans la proportion de 40 p. 100. Les métacercaires qu'elles hébergeaient, localisées surtout dans les muscles et la cavité générale, semblaient appartenir, pour la plupart, à l'espèce $P l$. medians; toutefois, nous n'avons pu confirmer ce diagnostic sommaire que pour les plus grandes métacercaires, atteignant dans leur kyste les dimensions suivantes : 450 à $500 \mu$ de diamètre, et, hors du kyste : $650-700 \mu \times 300-350 \mu$ (fig. 52).

Notons immédiatement qu'à l'inverse de Ch. Joyeux et ses collaborateurs qui, pendant l'hiver, n'ont plus trouvé chez les gammares que des kystes de mêmes dimensions, nous avons constaté au contraire de façon constante la présence simultanée, chez les larves de Sialis, de nombreux petits kystes sphériques d'un diamètre moyen de 150 à $200 \mu$ et de quelques grands kystes transparents semblables à ceux signalés ci-dessus.

Nous avons également disséqué un certain nombre de larves d'Aeschna grandis et quelques larves de Lestes sp. Ces insectes portaient fréquemment trois sortes de métacercaires : $P l$. medians, Prosotocus confusus, et les larves d'Aeschna présentaient, en outre, mais beaucoup plus rarement, une très grande métacercaire de près de $1 \mathrm{~mm}$., possédant une immense vessie dessinant un $\mathrm{U}$ très allongé atteignant presque la ventouse orale chez le distome sorti de son kyste; ce kyste était lui-même formé d'une membrane épaisse stratifiée et très réfringente. Nous avons examiné une trentaine de ces libellules pendant les mois d'hiver sans trouver de formes progénétiques de $\mathrm{Pl}$. medians. 
Entre le 15 décembre et le 15 mai, nous n'avons pas noté de progression sensible chez Sialis lutaria dans la taille des kystes de Pleurogenes medians; la proportion des grandes et des petites formes, soit environ un grand kyste pour cinq petits, est également demeurée la même. Ces observations confirment celles des auteurs qui ont étudié cette évolution (Joyeux, Neuhaus), selon lesquelles le développement pendant l'hiver subit une diapause. Nous n'avons pas observé non plus de progénèse naturelle, mais ce fait est peut-être imputable au printemps tardif et à la rigueur de la température.
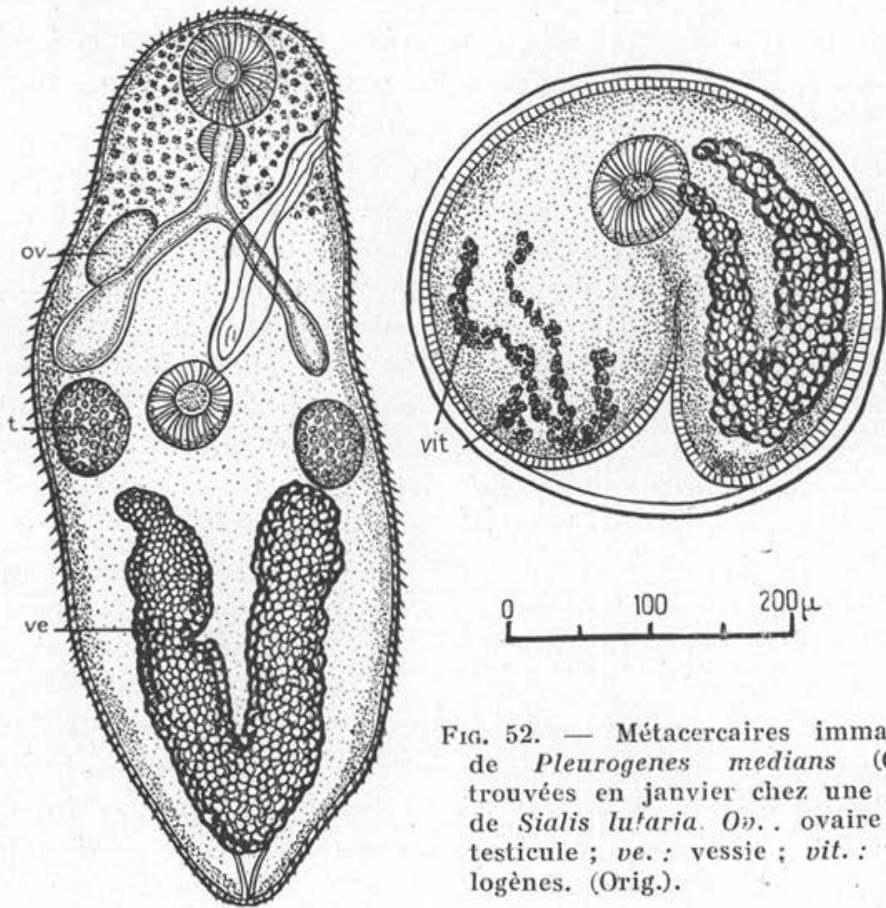

FIc. 52. - Métacercaires immatures de Pleurogenes medians (Olss.) trouvées en janvier chez une larve de Sialis Iutaria. O\#.. ovaire; $t$. : testicule ; ve. : vessie ; vit. : vitellogènes. (Orig.).

\section{Etude expérimentale}

Nos recherches ont porté sur les trois points suivants :

a) Evolution de métacercaires de Pl. medians chez un hôte spontanément infesté (la larve de Sialis lutaria), conservé pendant plus de cinq mois en captivité dans des conditions particulières de température et d'éclairement.

Interprétation de l'action de certains facteurs dans le déterminisme de la progénèse. 
b) Evolution des métacercaires de $P l$. medians chez un hôte expérimentalement infesté, la larve de phrygane.

Etude du cycle évolutif : cercaire virgule, enkystement; modalités de croissance de la métacercaire selon l'époque de l'infestation et l'âge de cette métacercaire ; évolution chez différents hôtes définitits connus ou nouveaux.

c) Conditions les plus favorables à la conservation de l'espèce ; leur concours éventuel au maintien de la progénèse chez $P l$. medians.

a) Eivolution de métacercaires de $P l$. medians CHEZ Un hôte SPONTANÉment Infesté (LA LARve de Sialis lutaria) CONSERVÉ EN CAPTIVITÉ

Ne connaissant pas la biologie des Sialis, et ne sachant pas, $\dot{a}$ priori, l'intérêt qu'elles représentaient pour l'étude du présent cas de progénèse, nous avons, d'abord, déposé des larves dans un grand cristallisoir de $30 \mathrm{~cm}$. de diamètre, contenant de l'eau de Seine au $p \mathrm{H}$ assez élevé, des herbes aquatiques et un grand nombre d'aselles, et des Bithynia assez abondantes, récoltées à notre gîte habituel. Nous ajoutions, une ou deux fois par semaine, une certaine quantité de larves de Chironomus plumosus pour nourrir les Sialis et même les Bithynia, toutes deux très voraces.

Cet essai n'a pas abouti ; en dépit de nos précautions, les larves de Sialis se sont entre-dévorées et les dernières ont été la proie des Bithynia. D'autre part, elles se cachent dans les herbes et sont alors difficiles à observer.

Nous avons alors isolé 16 exemplaires d'entre elles dans des tubes Borrel, avec des conferves et des aselles qui vivent dans le même milieu et leur servent de pâture. Ces tubes ont été placés dans une étuve où la température a été maintenue de façon constante, du 15 janvier au $1^{\text {er }}$ mai, aux environs de $25^{\circ} \mathrm{C}$. Du $1^{\text {er }}$ mai au $1^{\text {ter }}$ juin, l'étuve étant déréglée, la température inconstante a varié de $18^{\circ}$ à $25^{\circ}$. Cette étuve exposée au midi assurait aux individus en expérience le maximum d'éclairement, car nous avons eu maintes fois l'occasion de constater que l'action ménagée des rayons solaires avait un rôle important dans l'accélération du développement des formes larvaires de trématodes.

Huit de ces larves, sacrifiées au bout d'un mois, six semaines. deux mois et neuf semaines, n'ont pas présenté de formes progénétiques. Cependant, toutes, sauf deux, étaient parasitées; mais le développement des kystes ne devint manifeste qu'au bout de deux mois de séjour à l'étuve. Il y avait toujours coexistence de grandes 
et de petites formes, de tailles inégales, toutefois les premières atteignaient parfois 550 à $600 \mu$. Les grands kystes très transparents commençaient à se couvrir de taches brunes et de nombreux petits kystes étaient entièrement recouverts d'une croûte chitineuse, produit du métabolisme de l'hôte. Nous pensions alors que, dans cette enveloppe épaisse, le distome devait s'étioler et finalement mourir ; nous verrons plus loin que, loin de compromettre son développement, cette protection de l'hôte favorise au contraire l'évolution du distome.

Le 8 avril, nous trouvions chez deux nouvelles larves de Sialis, isclées depuis onze semaines, et sacrifiées (expériences 650 et 650/ I.A.B.), deux grands kystes chitineux, qui, en dépit de cette xénoformation, laissaient apparaître de nombreux œufs, visibles par transparence. Les dimensions de ces œufs étaient de $28 \times 18 \mu$.

L'extraction du distome hors de son kyste par les procédés habituels, pression, introduction entre lame et la lamelle de solutions hypotoniques ou hydrolysantes, nous a permis de constater la présence d'une triple enveloppe kystique : la première enveloppe, incrustée de mélanines qui lui donnent une apparence croûteuse et une coloration brunâtre, est, comme nous le signalions, un xénokyste ; elle se brise facilement. Les deux autres enveloppes, minces et transparentes, opposent une incroyable résistance à tous les moyens mécaniques ou chimiques utilisés pour libérer le trématode inclus. L'enveloppe la plus interne, en particulier, très plastique, se distend sans éclater sous la pression, et il nous est arrivé de casser la lamelle en appuyant trop fort, sans parvenir à rompre cette membrane. Les solutions hypotoniques ou acides pénètrent aussitôt à l'intérieur du kyste et détruisent le distome sans attaquer son enveloppe. La pepsine chlorhydrique produit le même effet, et nous avons eu, un jour, le cas d'un de ces kystes contenant un distome portant près de 200 œufs, qui, après action de suc gastrique artificiel, s'est trouvé vidé entièrement de son contenu, lysé par la pepsine ; seul demeurait la membrane intacte et les œufs ainsi mis en liberté dans le kyste.

Nous avons finalement opéré avec du suc gastrique naturel, ponctionné dans l'estomac d'un batracien adulte, milieu où se produit spontanément l'évolution de la métacercaire en adulte. Son action est lente et il faut agir en même temps mécaniquement sous le microscope, pendant une à deux heures, par pression douce sur la lamelle et déshydratation ménagée.

Les deux distomes contenus dans les kystes observés le 8 avril avaient produit environ 120 à 150 œufs chacun, dont une quinzaine 
avaient été pondus dans le kyste, phénomène entièrement nouveau dans ce cas de progénèse (voir page 137).

Rappelons que le maximum d'œufs observé jusqu'à présent dans la nature était de 64 chez des Gammarus pulex L., examinés par R.-Ph. Dollfus et récoltés par E. Brumpt au Bois de Boulogne, le 2 juin 1922.

Il y avait donc, chez les individus progénétiques développés chez ces larves de Sialis, une progénèse plus active et peut-être plus parfaite que chez ceux observés jusqu'à présent chez divers insectes ou crustacés, le déterminisme de la ponte dans le kyste résultant vraisemblablement d'une fécondité plus grande du distome.

Signalons toutefois, à côté de ces kystes chitineux progénétiques, la présence d'autres grands kystes, de mêmes dimensions, généralement transparents et non progénétiques.

Une autre larve de Sialis, morte le 20 avril (soit après douze semaines, plus quatre jours d'isolement), présentait environ quinze grandes métacercaires de Pleurogenes medians, dont trois seulement présentaient de 3 à 20 œufs.

Le 12 mai, nous avons disséqué trois nouvelles larves de Sialis, isolées au laboratoire. Elles portaient de très nombreuses petites métacercaires avec ou sans xénokystes. Une seule larve présentait trois grandes métacercaires progénétiques, dont deux avec enveloppe externe incomplètement chitinisée, tandis que le troisième kyste, recouvert d'incrustes, était entièrement opaque.

La première de ces métacercaires avait environ 50 œufs dans l'utérus et d'assez nombreux œufs (près de 20) étaient épars dans le kyste (fig. 53, B). Ces œufs paraissant bien formés et mûrs, nous les avons utilisés pour infester une trentaine de jeunes Bithynia tentaculata d'élevage (expérience 669/I.A.B.) et tenter ainsi un cycle abrégé ; mais nous n'avons pu constater l'éclosion des miracidiums.

La seconde métacercaire progénétique montrait un œuf parfait et deux autres atrophiés, réduits à de la substance coquillère.

La troisième avait huit œufs bien formés dans l'utérus mais aucun dans le kyste ; la vessie était de forme aberrante et assez semblable à celle de Prosotocus confusus, parasite fréquent des libellules au Bois de Boulogne. Néanmoins, sa triple enveloppe kystique, que nous n'avons pu réussir à rompre, ne nous a pas permis d'identifier ce distome avec certitude (fig. 53, A).

Le $1^{\text {er }}$ juin, une quinzième larve de Sialis a été sacrifiée par nous après cinq mois et demi de vie captive, dans les conditions expérimentales que nous avons relatées plus haut ( $n^{\circ}$ 675/I.A.B.). Cette larve manifestait depuis un mois un grand ralentissement de son 
activité. Elle ne réagissait plus aux agitations artificielles créées dans l'eau qui la baignait. Seul, le contact d'une pince éveillait encore chez elle une faible vitalité. Observons en effet qu'à cette époque, dans la nature, la larve est sortie de l'eau pour se cacher sous la terre et se métamorphoser en nymphe. Cette prolongation inhabituelle de sa vie larvaire ne peut sans doute se poursuivre très longtemps, à moins que l'état larvaire ne dure deux ans et que nos spécimens aient appartenu encore à la première année.

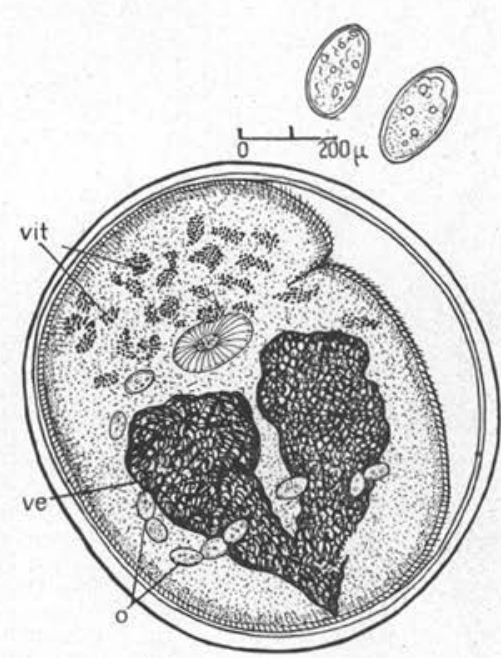

A

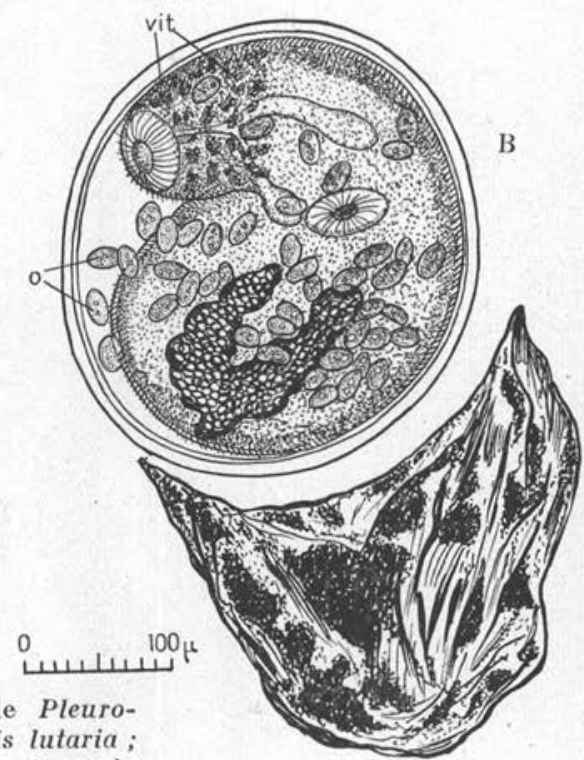

Fig. 53. - Métacercaires mùres de Pleurogenes medians d'une larve de Sialis lutaria; noter la ponte des oufs dans le kyste et la triple enveloppe kystique. A gauche, forme à vessie aberrante. (Orig.).

La larve 675 présentait dans les muscles et la cavité générale un très grand nombre de kystes de toutes dimensions, dont environ vingt étaient de grande taille (450-600 $\mu$ de diamètre). Sur ces vingt kystes, quinze étaient fortement chitinisés et tous progénétiques; les cinq autres, sans protection mélanique, ne présentaient pas d'œufs.

Sur les quinze métacercaires progénétiques, six montraient au moins 200 œufs et peut-être davantage; les autres en avaient un nombre variant de $2-3$ à 75 ou 100 . Ces kystes ne présentaient en général que deux enveloppes, la plus interne étant toujours élastique et d'une extrême solidité, la membrane externe, encroûtée de mélanines, étant au contraire, par simple compression, assez facile 
à briser sans léser le distome. Nous n'avons pu faire éclater que la membrane interne de distomes ne présentant encore que très peu d'œufs, qui nous ont permis néanmoins de les identifier de façon certaine à Pleurogenes medians (fig. 54).

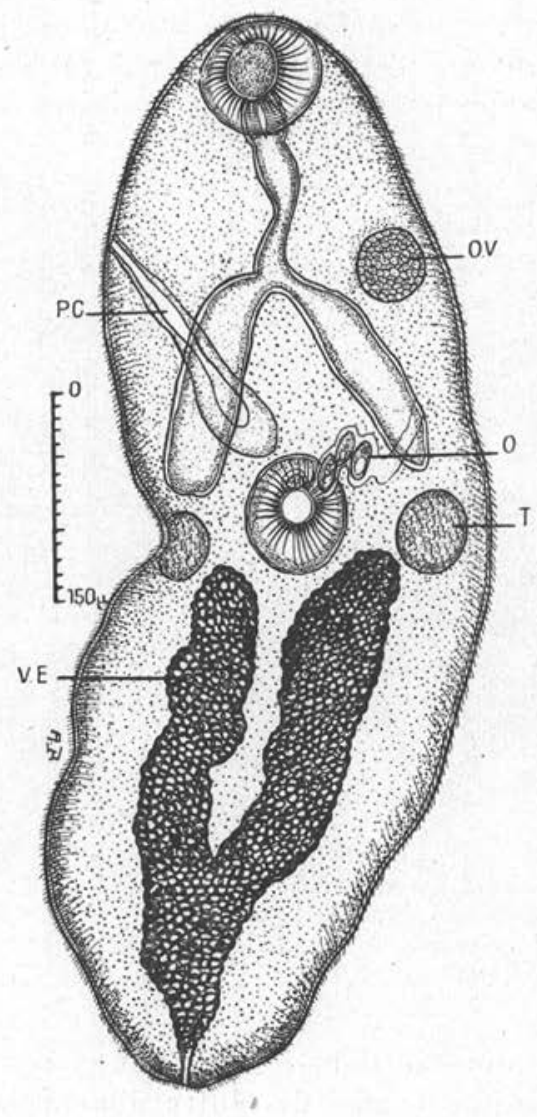

Fı́. 54. - Métacercaire progénétique de Pleurogenes medians, sortie de son kyste vue par la face dorsale et provenant d'une larve de Sialis lutaria. O. : cufs ; OV.: ovaire ; P.C. : poche du cirre; T. : testicule ; V.E. : vésicule excrétrice. (Orig.).

Les métacercaires ayant produit 200 œufs, en avaient beaucoup (une cinquantaine) répandus dans le kyste. Ces œufs n'étaient pas tous bien formés : ceux qui se trouvaient dans les premières anses utérines étaient parfois réduits à du matériel vitellin entouré de substance coquillère, ou encore d'une taille nettement inférieure à 
la normale ( $20 \mu$ au lieu de 28 ). Enfin, un tiers environ des œufs pondus dans le kyste étaient collapsés ou plus ou moins dégénérés. En revanche, les œufs de dimensions normales $(28 \times 18 \mu)$, en particulier ceux qui se trouvaient dans la seconde moitié de' l'utérus, dans le voisinage du pore génital, paraissaient mûrs.

Nous avons retrouvé ces particularités chez la plupart des métacercaires ayant pondu de nombreux œufs dans leur kyste, et hébergées par la larve de Sialis 675/A.B.-I.

Nous concluons de ces observations qu'il faut certainement admettre une action de la température dans le déterminisme de la progénèse chez Pleurogenes medians. Nous avons en effet observé nos premières métacercaires progénétiques le 8 avril, date à laquelle nous n'avons pas trouvé de progenèse dans la nature, lors de nos prospections périodiques au Bois de Boulogne.

Le facteur éclairement joue peut-être également; son rôle paraît moins net. La larve de Sialis vit dans la vase ; dans les conditions expérimentales, les rayons solaires l'atteignaient plus aisément, mais toujours à travers les végétaux aquatiques où elle se dissimule.

Y a-t-il une action de l'hôte? Nous avons obtenu au laboratoire, chez la larve de Sialis, un nombre d'œufs infiniment plus grand que ceux précédemment observés chez le gammare dans la nature. D'autre part, les observations de Ch. Joyeux et de ses collaborateurs avaient constaté la dégénérescence des métacercaires spontanées portées par les gammares gardés pendant l'hiver en captivité, dans des conditions expérimentales assez voisines des nôtres. Nous ne saurions néanmoins conclure à une action de l'hôte avant que de nouvelles observations sur l'évolution des kystes de Pleurogenes medians chez le gammare en captivité ne confirment les premiers résultats publiés sur ce sujet. Il est possible que la température n'intervienne qu'en association avec les rayons de la lumière solaire, ou tout autre facteur extérieur passé inaperçu.

Toutefois, signalons que ni Ch. Joyeux et ses collaborateurs, ni R.-Ph. Dollfus n'ont observé chez le gammare de kystes mélaniques. Dollfus écrit en effet en 1924 : "Examinés in vivo, ces kystes étaient sphériques ou un peu ovales, à paroi incolore, transparente, très mince, quelle que soit leur taille. "Dans nos expériences, au contraire, nous avons toujours observé chez la larve de Sialis lutaria cette enveloppe pigmentaire, particulièrement dense sur les kystes renfermant de nombreux oeufs, tandis que les kystes transparents ne montraient jamais d'œufs.

W. Neuhaus (1940), étudiant avec beaucoup de détail le processus de l'enkystement de la cercaire virgule de $P I$. medians chez différentes larves d'insectes est le seul à mentionner cette forma- 
tion de mélanine, de même que la présence des trois membranes kystiques que nous avons si souvent observées. Voici comment il décrit leur formation progressive :

« L'enkystement est probablement déclenché par les propriétés chimico-physiques du liquide cœlomique de l'hôte et, par suite, ne peut être considéré que partiellement comme un comportement autonome des cercaires. Il se forme d'abord une très mince pellicule incolore. Celle-ci est identique à la couche cuticulaire externe de la cercaire. J'ai pu parfois observer directement sa séparation de la paroi interne de la cuticule. A la limite des deux couches, on observe de fines granulations provenant de la couche inférieure et peut-être dues à la disparition de la couche cuticulaire moyenne. La formation ultérieure du kyste se réalise par l'intermédiaire de la ventouse buccale. Sous l'effet des mouvements du corps, une substance d'abord granuleuse, qui s'homogénéise ensuite, est évacuée par la ventouse orale et forme la paroi kystique (fig. 55). Cêtte substance est produite par

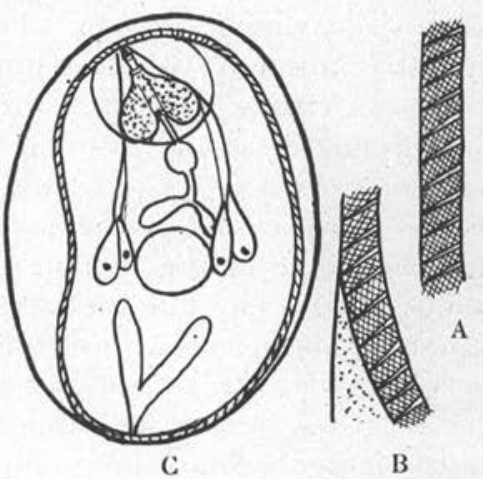

FIG. 55. - Processus de décollement de la cuticule de la cercaire pour la formation de la première enveloppe kystique. A, cuticule avant l'enkystement; B, décollement de la cuticule et présence de fines granulations ; C, kyste formé (d'après W. Neuhaus, 1940). les organes piriformes. Peut-être la sécrétion des glandes de pénétration contribue-t-elle aussi à l'élaboration de la membrane kystique. Celle-ci, achevée, est optiquement homogène, incolore et d'une épaisseur moyenne de 2-4 $\mu$. Par de légères pressions sur un kyste sous lamelle, on voit que le kyste se compose de deux couehes. L'enkystement apparaît ainsi comme une dermatisation modifiée. Pendant dix à quatorze jours, les kystes demeurent sans changement. Puis l'hôte forme progressivement autour de cette double membrane une paroi conjonctive ; au bout de plusieurs semaines, celle-ci peut devenir assez importante ; pendant les premiers temps, elle demeure à peu près incolore. Plus tard, la partie formée la première, en contact avec l'autokyste, devient brunâtre, probablement par formation de mélanine. Le kyste se compose finalement de trois couches. Les hôtes intermédiaires ne réagissent pas tous également aux parasites. Les couches adventices sont, par exemple, plus minces chez les larves d'Aeschna que chez celles de Libellula. » 
Nous ne saurions prétendre que ces formations mélaniques constituent un milieu particulièrement favorable à la progénèse, étant donné que Neuhaus, qui a étudié avec tant de soin l'élaboration de ces kystes, n'a jamais observé de métacercaires progénétiques de $\mathrm{Pl}$. medians. Notons tout au moins que cette triple barrière osmotique ne constitue pas un obstacle au développement et à la croissance du ver. Nous avons déjà signalé avec quelle rapidité toute substance non isotonique agissait sur le distome. L'adaptation à la vie osmotique semble ici parfaite et doit permettre l'utilisation au maximum des sucs nutritifs de l'hôte.

Nous avons abordé l'étude de l'action de certains milieux nutritifs sur le développement du distome enkysté (voir technique, p. 64-65). Cette étude est encore inachevée; et nous ne pouvons relater ici que l'action du milieu Galliard (eau ordinaire, gélose, glycose pur et chlorure de sodium) qui nous a donné en trois semaines un accroissement notable des métacercaires ensemencées incluses dans les tissus de l'hôte et âgées de trois semaines.

\section{b) Evolution des métacercaires de $\mathrm{Pl}$. medians}

CHEŻ UN HôTE EXPÉRIMENTALEMENT INFESTÉ (LA LARVE DE PHRYGANE)

P. Mathias (1924), Ch. Joyeux et ses collaborateurs (1930), enfin W. Neuhaus (1940), ont étudié le cycle évolutif de Pleurogenes medians :

Mathias, faisant évoluer des métacercaires de grande taille, mais non progénétiques, chez Rana temporaria et Hyla arborea, a obtenu en douze jours, dans l'intestin grêle de ces animaux, des vers adultes présentant de nombreux œufs.

Neuhaus (1940 et 1941) a étudié expérimentalement ce cycle en infestant des larves d'Aeschna, Libellula et de Phryganea, avec la cercaire virgule de Pleurogenes medians, émise par des Bithynia tentaculata. Il a observé que les métacercaires étaient déjà infestantes au bout de quarante-huit heures et le restaient jusqu'à 67 jours, et que la durée totale du cycle variait entre 88 et 138 jours (fig. 56).

Recherchant dans la nature le pourcentage d'infestation des Bithynia, il admet que l'infestation naturelle se produit en été, que le maximum d'infestation s'observe en automne, époque à laquelle les mollusques présentent à la fois des infestations anciennes et récentes. Pendant l'hiver, l'évolution et les infestations cercariennes sont suspendues; au printemps, les métacercaires jeunes atteignent leur taille maximum; les mollusques les plus infestés périssent, de sorte que le pourcentage total d'infestation baisse, les infestations nouvelles ne s'étant pas encore manifestées. 
D'après Joyeux et coll. (1930), qui se sont attachés surtout à la durée d'évolution de la métacercaire jusqu'au stade progénétique, l'infestation des gammares se produirait en juin ; notons que l'opi-

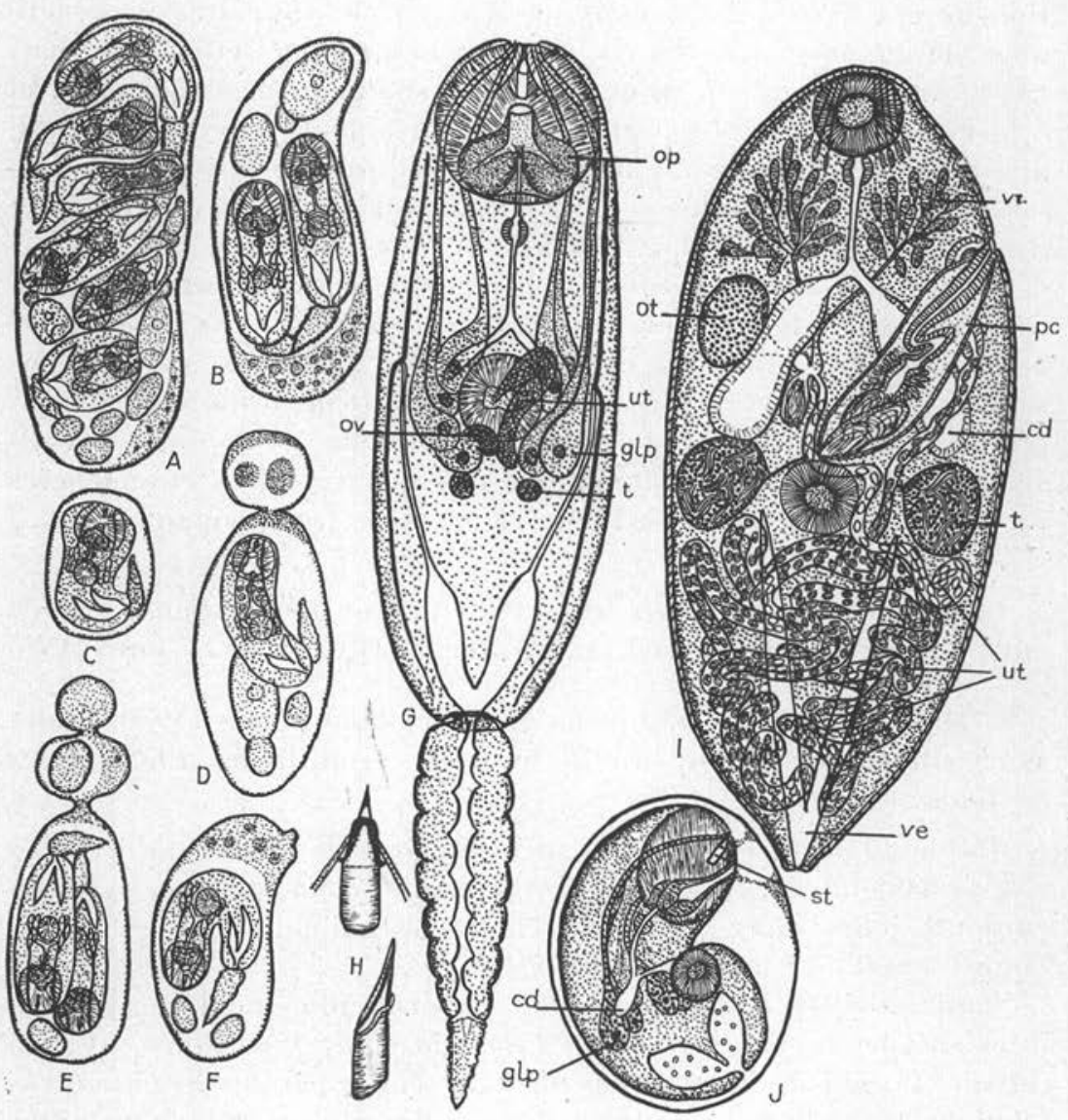

Fig. 56. - Evolution de Pleurogenes medians (Olss.) : A, B, C, D, E, F, sporocystes ; G, cercaire virgule ; H, stylet: J, métacercaire ; I, adulte ; $c d$ : cacum digestif ; $g l p$ : glandes de pénétration : $o p:$ organe piriforme ; ov : ovaire ; $p c$ : poche du cirre ; st: stylet; $t$ : testicules ; ut: utérus ; ve : vésicule excrétrice; $n t$ : vitellogènes (d'après W. Neuhaus, 1940)

nion de ces auteurs est fondée sur des statistiques d'infestation naturelle, non sur des infestations expérimentales. Ils admettent ainsi que la croissance se poursuit pendant l'été et l'automne, les organes évoluent pendant la diapause hivernale, l'activité sexuelle se manifeste au printemps et la progénèse apparaîtrait peu après. 
Selon ces auteurs, la progénèse semblerait donc un état ultime du stade métacercaire, qui ne se manifesterait pas nécessairement, mais qui, suivant immédiatement la période d'activité génitale de la métacercaire (constatée sur coupes), en apparaîtrait comme la suite logique.

Nous avons été surprise par la longue durée d'évolution signalée par ces chercheurs pour la croissance de la métacercaire chez ses différents hôtes intermédiaires. C'est pourquoi nous nous sommes proposée d'étudier au laboratoire son développement chez un hôte expérimental neuf et signalé par Neuhaus comme très favorable, la larve de phrygane.

Nous avons commencé par récolter un grand nombre de Bithynia tentaculata du gîte du Bois de Boulogne. Certaines ont émis des cercaires en pleine saison d'hiver ( 15 janvier), ce qui prouve que l'infestation des larves d'insectes et crustacés, même ralentie par la température, se produit constamment. Nous en avons autopsié environ 150 , parmi lesquelles 12,3 p. 100 étaient infestées par une cercaire virgule. Des larves de phryganes, placées avec les débris d'hépatopancréas et du corps du mollusque, ont montré, au bout de quarante-huit heures, quelques métacercaires type Pleurogenes medians.

Nous avons également isolé des Bithynia positives et essayé d'infester avec leur cercaires un certain nombre de larves d'insectes (larves de phryganes, larves de Chironomus plumosus, larves de Stegomyia fasciata); seules les larves de phryganes se sont infestées. Neuhaus signale également la rareté de l'enkystement de cette cercaire chez la larve de chironome. Observons que les Bithynia tentaculata hébergent un grand nombre de cercaires du type virgula. A Richelieu (Indre-et-Loire), où nous en avons trouvé un certain nombre, la cercaire virgule de Pleurogenes medians existe, mais nous n'avons jamais vu de métacercaires progénétiques. L'identification de ces cercaires virgules est assez malaisée si l'on veut s'en tenir à la morphologie de la cercaire ou de la métacercaire qui vient de s'enkyster. Il faut donc faire appel également aux propriétés biologiques des formes larvaires et attendre d'autre part que la métacercaire soit suffisamment évoluée pour permettre une identification certaine. C'est ainsi que la spécificité de la cercaire pour le second hôte intermédiaire fournit souvent une bonne indication. Nous donnons ci-dessous la figure curieuse d'un enkystement multiple d'une cercaire virgule émise par Bithynia tentaculata, à Richelieu (fig. 57).

Cet enkystement insolite et curieux s'est produit sur une nymphe de Chironomus plumosus; il était accompagné par la

ANN. de Parasitologie, T. XXVI, N ${ }^{\circ} 3 .-1951$. 
pénétration de quelques rares cercaires à l'intérieur du corps de cet animal. Peut-être s'agit-il de la cercaire de Pleurogenes medians qui, en l'absence d'un hôte plus favorable, se serait enkystée d'une manière anormale. La nymphe de chironome, qui ne peut être conservée bien longtemps en captivité, ne nous a pas permis de suivre l'évolution de ces métacercaires.

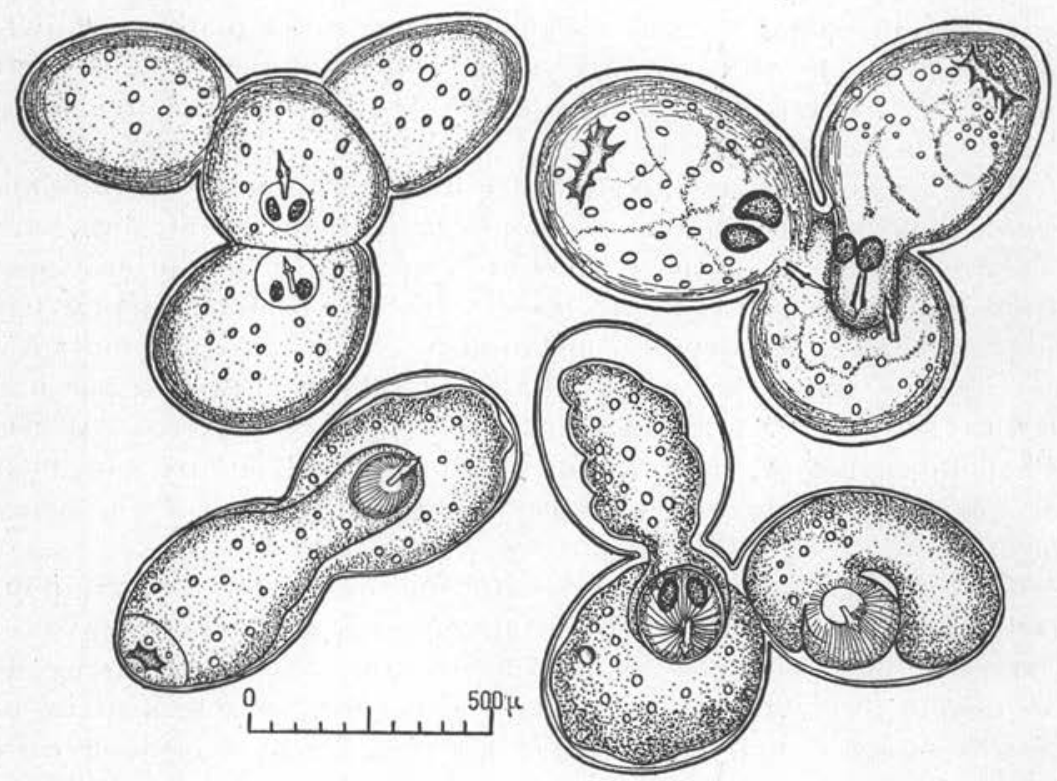

FIG. 57. - Enkystement multiple de cercaires virgules de Bithynia tentaculata chez un hôte peu favorable, la nymphe de Chironomus pltimosus. Noter la persistance du stylet et des organes virgules. (Orig.).

C'est dans une grande cuve de verre de $80 \mathrm{~cm}$. de long sur $40 \mathrm{~cm}$. de large et $30 \mathrm{~cm}$. de haut que nous avons placé, le 21 mars dernier, environ 150 Bithynia tentaculata prélevées au Bois de Boulogne et 50 larves de Phryganea sp. provenant d'un gîte de la station de Richelieu, où Pleurogenes medians semble ne pas exister. En effet, la dissection de 25 larves témoins de même origine a confirmé l'absence de toute métacercaire chez cet hôte intermédiaire. Cette cuve, placée dans une pièce chauffée, était exposée au midi.

Des examens ont été pratiqués tous les quinze jours; au bout des deux premières semaines, les larves examinées présentaient en moyenne 6 à 10 métacercaires d'environ $150 \mu$ de diamètre 
par larve. Ces dimensions n'ont guère varié après un mois et même six semaines. A partir du 15 mai, la croissance des kystes est devenue manifeste et le $1^{\text {er }}$ juin dernier, soit après quinze semaines d'infestation, la plupart d'entre eux mesuraient 300 à $350 \mu$ dans leur plus grand diamètre. Cependant, quelques petits kystes étaient égalements présents, témoignant d'une infestation plus récente. Les dernières larves examinées présentaient de 15 à 30 métacercaires par individu.

Nous avons pu comparer le temps d'évolution de la métacercaire chez l'hôte définitif, selon que cette métacercaire est jeune ou âgée. Chez Rana temporaria, le développement de grands kystes non progénétiques, trouvés spontanément chez la larve de: Sialis lutaria, s'effectue en 10 à 13 jours (expérience 616/A.B.-I) : les distomes trouvés dans l'intestin grêle moyen ont de nombreúx œufs dans leur utérus. Des métacercaires de larves de phrygane, obtenues expérimentalement 30 jours après exposition des larves aux cercaires, ne sont, au bout de 15 jours, que de petits vers immatures de 400 à $500 \mu$ de long dans l'intestin grêle de la même Rana temporaria. Ces expériences ont été effectuées à une température oscillant autour de $20^{\circ} \mathrm{C}$.

L'évolution de ces métacercaires de 30 jours chez la larve de phrygane a été étudiée chez Chamæleon vulgaris. Deux jours après ingestion, les vers étaient simplement sortis de leurs kystes mais se trouvaient déjà localisés dans l'intestin moyen de ce reptile. Au bout de 15 jours, les vers avaient notablement grandi ; ils n'avaient pas quitté leur habitat dans l'intestin grêle antérieur et surtout moyen. Après 30 jours, les vers, que l'on retrouve toujours au même endroit, montraient des centaines d'œufs mûrs dans l'utérus (expériences 667,667 ', 668, 668', 679, 680, 681 et 682/A.B.-I). Ces expériences, réalisées du 11 mai au 10 juin, nous ont permis de vérifier que Pleurogenes medians, parasite habituel de batraciens, évoluait parfaitement chez un reptile du genre Chamæleon (1) et que, chez cet animal, l'évolution du parasite au printemps est rapide puisqu'elle s'est effectuée en un mois, ce qui porte le développement total à 60 jours, Neuhaus, en effet, estime de trois mois et demi à quatre mois et demi le temps total d'évolution jusqu'au ver adulte.

Nous avons essayé l'évolution chez un autre reptile, Lacerta viridis, ce lézard est malheureusement mort trois jours après son infestation. Nous avons retrouvé de petits vers immatures dans l'intestin moyen; ils étaient sortis de leur kyste, mais, en raison

(1) Signalons qu'un caméléon témoin autopsié était indemne de parasites, et que parmi les huit autres, un seul (682), a présenté dans l'intestin deux grands trématodes, non déterminés, mais d'espèce différente de $\mathrm{Pl}$. meedians. 
de l'extrême chaleur, le lézard, mort peut-être depuis une heure, commençait à sentir, c'est sans doute pourquoi les vers étaient morts et certains déjà dégénérés (expérience 673/A.B.-I).

L'évolution chez Anguis fragilis a été tentée (exp. 673'/A.B.-I), mais n'a pas été suivie de succès ; l'autopsie de l'animal, six semaines après son infestation par 25-30 métacercaires de 40 jours, provenant de larves de phryganes infestées expérimentalement, n'a pas permis de retrouver un seul de ces distomes dans le tube digestif.

Chez Discoglossus pictus, l'ingestion par l'un de ces batraciens (dont 4 spécimens témoins s'étaient montrés indemnes de parasites) de 5 métacercaires jeunes ( 8 jours), obtenues expérimentalement chez des gammares, a permis de retrouver 15 jours plus tard, dans l'intestin moyen, 2 exemplaires immatures de Pleurogenes medians (exp. 683/A.B.-I). Cet amphibien n'avait pas encore été signalé comme hôte définitif expérimental de $P l$. medians.

Chez Triton palmatus (651/I.A.B.), l'évolution est semblable à celle de Rana, et le développement des métacercaires âgées, mais non progénétiques, provenant de larves de Sialis lutaria, s'effectue en 12 à 15 jours.

Neuhaus a effectué également un grand nombre d'expériences sur l'évolution comparée chez l'hôte définitif de métacercaires expérimentales plus ou moins évoluées ; il admet que le développement chez l'hôte définitif dépend du temps de séjour de la métacercaire chez l'hôte intermédiaire. Le développement morphologique du distome reste néanmoins semblable, que l'infestation de l'hôte définitif ait eu lieu à un stade jeune ou avec des kystes âgés. Cependant, le temps total de l'évolution n'est pas toujours constant; il est en principe de trois mois et demi, mais il peut atteindre parfois quatre mois et demi ; ces différences sont vraisemblablement dues à certains facteurs extérieurs, nutrition, état physiologique de l'hôte, température, etc.

Etant donné que l'infestation de l'hôte définitif peut se faire avec des métacercaires de 2 à 117 jours, et que, néanmoins, leur développement morphologique reste le même, il faut bien admettre que ce distome trouve, pendant un certain temps tout au moins, les mêmes conditions de développement dans la cavité générale et les téguments de l'insecte, son hôte intermédiaire, que dans l'intestin moyen des amphibiens, ses hôtes définitifs.

Il y a donc chez Pleurogenes medians une certaine plasticité, une spécificité moins stricte, lui permettant de s'adapter à des genres de vie très différents : vie osmotique dans le kyste, vie libre dans le tube digestif, correspondant à des conditions de nutrition également très diverses. Cette plasticité se manifeste encore par l'évolution 
facile du ver chez des hôtes définitifs, appartenant à des classes différentes, comme les batraciens et les reptiles.

Pourquoi ne pas admettre dès lors que, l'activité génitale du distome se manifestant déjà parfois dans le kyste, la fécondation et la ponte puissent s'y produire également? Cette éventualité s'appuyant sur la remarquable faculté d'adaptation de ce trématode, n'aurait, à priori, rien d'invraisemblable.

La progénèse a été rarement signalée en dehors du gìte découvert par Mathias. A Richelieu (Indre-et-Loire), où ce parasitisme est très commun, nous n'avons jamais observé de progénèse et de nombreux chercheurs avant nous n'ont pas été plus heureux. Cependant, R.-Ph. Dollfus signale en avoir vu chez des gammares récoltés dans la Veude (Indre-et-Loire) (1). En Allemagne, les belles études de Neuhaus, effectuées avec un soin extrême et une grande rigueur, sur un matériel abondant, ne mentionnent aucun cas de progénèse ; néanmoins, cet auteur parle de métacercaires ayant séjourné de 2 à 117 jours chez leur hôte intermédiaire.

Il est vrai que Neuhaus a fait ses expériences, non sur des gammares, mais sur des larves de libellules et de phryganes, hôtes peut-être peu favorables au déterminisme de la progénèse. D'autre part, la durée des infestations expérimentales de cet auteur était peut-être insuffisante pour permettre à ce phénomène de se manifester (2) ; car, il semble bien que le temps joue un rôle important dans l'apparition de la progénèse chez Pleurogenes medians; il faudrait évidemment, pour en être sûr, pouvoir conserver à une température moyenne de $18-20^{\circ}$, pendant $10-12$ mois, des larves jeunes de Sialis indemnes de parasites, expérimentalement infestées, et faire des examens périodiques de leurs métacercaires.

L'action de l'hôte, la durée d'infestation, la température, sont-elles des facteurs suffisants, et n'y a-t-il pas, à l'origine de la progénèse chez $P$. medians, un facteur génétique, inscrit ou non dans le patrimoine héréditaire de telle ou telle souche, qui expliquerait l'inconslance du phénomène, non seulement dans les différents gîtes, mais à l'intérieur d'un même hôte ?

La mise en évidence de ce caractère demanderait de patientes expériences que nous avons entreprises, mais que nous n'avons pu mener à bien, parce que nous avons manqué de matériel vivant, et plus particulièrement de larves neuves d'insectes : c'est ainsi que,

(1) Voir Extr. C. R. Acad. Sci., t. 207, 1938, p. 431.

(2) 2 gammares neufs sur 15 , exposés aux cercaires de $P$. micdians, en juillet 1950 , présentèrent, 75 jours plus tard, des métacercaires de grande taille, à double enveloppe kystique et aux vitellogènes très développés, mais non pro-* génétiques (706/A.B.-I). 
à partir d'une larve de Sialis, nous avons isolé une dizaine de métacercaires progénétiques et les avons fait ingérer à une Rana temporaria en vue d'obtenir des œufs ayant une maturation parfaite. Douze jours plus tard, cette grenouille a été sacrifiée et dans l'intestin grêle nous avons retrouvé huit distomes adultes. Avec les œufs de chacun d'eux pris isolément, nous avons infesté un lot de dix jeunes Bithynia tentaculata d'élevage. Nous nous trouvions ainsi avoir huit lots de dix $B$. tentaculata infestés chacun à partir des œufs d'une métacercaire progénétique mûris chez son hôte définitif.

Si ces prosobranches, dont nous n'avons pu suivre la destinée, avaient émis des cercaires, nous aurions infesté, avec chacun des lots, un certain nombre de larves neuves de phryganes et de Sialis, de manière à avoir autant de lots de larves infestées que de lots de Bithynia, ceci afin de suivre l'évolution des métacercaires expérimentales obtenues à partir d'une seule métacercaire progénétique. Les métacercaires expérimentales de chaque lot seraient donc toutes issues de la même souche, souche ayant manifesté le phénomène de la progénèse ; si toutes ces métacercaires étaient devenues progénétiques, ce serait, sans doute, que la progénèse est bien ici le fait d'un facteur héréditaire. Si une partie seulement des métacercaires étaient devenues progénétiques à l'intérieur d'un même lot, c'est peut-être que, dans la forme souche, la manifestation de la progénèse n'était que phénotypique. Supposons, en effet, que le gène soit dominant, on ne peut savoir à priori si une métacercaire progénétique est homozygote ou hétérozygote pour ce gène. Si elle est hétérozygote, on comprendrait qu'elle puisse donner naissance, dans les conditions que nous avons relatées, à des métacercaires progénétiques ou non. De même, si à l'intérieur d'un ou de plusieurs lots toutes les métacercaires devenaient progénétiques, on pourrait admettre que la métacercaire progénétique souche était homozygote pour le gène.

Des expériences témoins, effectuées dans les mêmes conditions avec un certain nombre de métacercaires âgées de 12 mois, mais non progénétiques et de la même espèce, permettraient de contrôler si, au bout de 8 à 12 mois, les métacercaires expérimentales obtenues restaient bien non progénétiques.

\section{c) Conditions les plus favorables}

A LA CONSERVATION DE L'ESPÈce ET AU MAINTIEN DE LA PROGÉNÈsE

La métacercaire progénétique est-elle capable d'assurer elle-même la pérennité de sa souche ou doit-elle être ingérée par un hôte définitif pour obtenir ce résultat? 
S'il faut l'intervention d'un hôte définitif, observons que contrairement à certains batraciens exotiques qui se nourrissent de poissons (Xenopus, Rana exotiques, etc...), les hôtes abritant le plus communément le ver adulte de Pleurogenes medians (Rana esculenta, $R$. temporaria, $R$. agilis, Hyla arborea, etc...) ne se nourrissent jamais dans l'eau. Dans ces conditions, les gammares hébergeant les formes progénétiques ne peuvent être ingérés et constitueraient une impasse s'ils n'étaient pas avalés par quelque triton vorace. Cet animal mange, en effet, certainement dans la nature des aselles et des gammares, puisqu'il présente parfois dans son corps des échinorhynques évoluant chez ces crustacés. Nous n'avons pas trouvé de tritons au cours de nos investigations au Bois de Boulogne; il est probable qu'ils n'y sont pas très abondants.

La larve de Sialis lutaria est-elle plus favorable à la conservation de l'espèce ? Il nous paraît très vraisemblable, en raison de la durée prolongée de son stade larvaire et du développement exceptionnel atteint chez cet hôte par la métacercaire de Pleurogenes medians, que la larve de Sialis lutaria permette aux formes progénétiques de se suffire à elles-mêmes, sans le secours d'un hôte définitif. Toutefois, la présence de ce dernier est sans doute nécessaire, le cycle progénétique restant probablement un phénomène assez rare. Cet hôte définitif pourra être le triton, comme pour le gammare, si l'insecte infesté est ingéré pendant son stade larvaire. Si le parasitisme persiste chez l'imago, il n'y aura pas d'obstacles à ce qu'il soit absorbé par les hôtes définitifs habituels de Pleurogenes medians (Rana, Hyla, Bufo). Nous avons obtenu sans difficulté chez Rana temporaria l'évolution de métacercaires hébergées par Sialis lutaria durant son stade larvaire.

Cet insecte discret, que sa biologie larvaire expose facilement, dans les fonds vaseux, aux attaques des cercaires virgules émises par les Bithynia, joue donc peut-être un rôle considérable et insoupçonné dans la conservation de la progénèse chez Pleurogenes medians. Il resterait à prouver que sa présence est constante et non d'apparition récente ou occasionnelle, et qu'il s'agit bien ici, comme l'admet R.-Ph. Dollfus (1924) dans le cas de l'enkystement chez le gammare de Pleurogenes medians, d'une adaptation évolutive durable et non d'une adaptation accidentelle et temporaire.

\section{Conclusions}

Avec Pleurogenes medians, nous avons abordé un cas de progénèse inconstante. A côté de l'évolution normale, de beaucoup la plus fréquente, apparaissent occasionnellement des formes progénéti- 
ques. Celles-ci ne représentent, en somme, que des métacercaires un peu plus avancées dans leur développement. Parvenues chez un hôte définitif, elles y achèvent normalement leur évolution et acquièrent ainsi rapidement leur maturité parfaite.

Nous avons montré le rôle probable de facteurs extérieurs tels que température, éclairement, métabolisme de l'hôte dans la manifestation de ce phénomène. Neuhaus, bien que n'ayant pas observé de progénèse, admet l'action de certains de ces facteurs sur la croissance et le développement morphologique du distome. Il n'y aurait donc rien de surprenant à ce que, chez les formes progénétiques, ces facteurs interviennent également pour permettre à la progénèse d'atteindre, chez la métacercaire, une expression plus complète. C'est bien ainsi que nous interprétons le développement exceptionnel de la progénèse chez les métacercaires progénétiques hébergées par la larve de Sialis lutaria, où les dimensions du kyste atteignent $650-700 \mu$ et le nombre des œufs 200 , dont plusieurs pondus dans le kyste.

Nous avons signalé d'autre part, au cours de son existence métacercarienne, la curieuse plasticité physiologique de Pleurogenes medians, qui peut poursuivre son développement, aussi bien chez des larves d'insectes ou de crustacés que dans l'intestin moyen d'amphibiens ou même de reptiles (expériences 616, 651, 667, 667', 668,668 ', 668”, 673, 673', 673'/A.B.-I). Cette grande faculté d'adaptation implique-t-elle une prédisposition particulière à la progénèse ? Nous pensons plutôt qu'elle explique la diversité de ses manifestations, depuis les formes où la progénèse, à peine amorcée, ne se signale que par la présence de coques plus ou moins atrophiées renfermant seulement du matériel vitellin, jusqu'aux métacercaires fécondes, émettant de nombreux œufs dont certains sont déjà répandus dans l'enveloppe kystique. Les divers degrés de progénèse que l'on peut observer résultent en quelque sorte des interactions particulières existant entre le distome et le milieu dans lequel il vit.

Mais le rôle initial, déterminant dans la progénèse, revient probablement ici encore au patrimoine héréditaire. On peut se demander qu'elle est la nature véritable de ce déterminant : s'agit-il seulement d'une sensibilisation de la souche à la progénèse, ce qui expliquerait l'inconstance du phénomène chez des métacercaires apparemment du même âge, ou bien encore de la mutation d'une souche déterminée fixée par la sélection? Dans ce dernier cas, l'isolement d'une souche homozygote pour ce gène permettrait peut-être d'obtenir, comme dans le cas de Paralepoderma brumpti ou de Ratzia joyeuxi, des métacercaires qui, toutes, obligatoirement, deviendraient progénétiques. 
Toutefois, cette progénèse, même obligatoire, et c'est ce qui fait l'intérêt de ce cas, reste encore imparfaite ; elle présente des degrés variables d'évolution qui, jusqu'à preuve du contraire, requièrent le concours d'un hôte définitif pour aboutir à une complète maturité. Mais, alors que dans les cas précédents rien ne peut intervenir pour modifier le cours du développement de la progénèse, il semble que, dans ce cas, divers facteurs puissent agir et déterminer peut-être, dans certaines conditions, une forme de progénèse parfaite : ainsi se trouverait réalisée la coexistence d'un cycle évolutif complet et d'un cycle abrégé, ce dernier restant conditionné par le milieu extérieur. Ce cycle nous paraît bien près de devenir effectif si les larves ou adultes de Sialis lutaria conservent les métacercaires de Pl. medians un temps suffisant pour permettre à leurs œufs de mûrir pendant la belle saison.

C'est qu'il faut, en effet, tenir compte de ce dernier facteur, le temps d'évolution. Toutes les observations expérimentales s'accordent à reconnaître que la durée du développement, chez Pleurogenes medians, est longue : elle s'étend sur plusieurs mois, avec une diapause hivernale si l'infestation s'est produite pendant l'arrièresaison. Précisons encore que ce facteur « durée » ne suffit pas à lui seul à faire naître la progénèse, pas plus que les conditions avantageuses de nutrition. Les métacercaires de Strigéidés, à très longue évolution, ne deviennent jamais progénétiques, pas plus que celles d'Opisthioglyphe ranæ ou de Leptophallus nigrovenosus dont les kystes très développés persistent chez les têtards pendant plus d'une année, mais n'ont jamais manifesté la moindre tendance à la progénèse. Ce phénomène n'est certainement pas un caractère acquis, malgré sa nature fluctuante et sa sensibilité particulière aux conditions du milieu dans lesquelles il se développe.

La progénèse de Pleurogenes medians apparaît comme une forme de transition entre l'évolution normale et celle des espèces parfaitement adaptées à ce processus simplifié de reproduction. Elle reste, de ce fait, encore malléable, et ses manifestations à venir ne seront redevables à la sélection que de ce que celle-ci aura fixé, dans le milieu naturel, qui soit favorable à leur épanouissement : préservation de certains hôtes, pérennité du gîte où ces hôtes se développent ; climat précocement tempéré, assurant à la métacercaire un temps d'évolution suffisant pour permettre la pleine réalisation de ses tendances progénétiques.

(à suivre).

Institut de Parasitologie de la Faculté de Médecine de Paris et Station expérimentale de Richelieu (Indre-et-Loire) (Dir.: Prof. H. Galliard) (Dir. honor. : Prof. E. Brumpt) 